\title{
Improved fractional Poincaré type inequalities on John domains
}

\author{
María Eugenia Cejas, Irene Drelichman and Javier C. Martínez-Perales
}

\begin{abstract}
We obtain improved fractional Poincaré inequalities in John domains of a metric space $(X, d)$ endowed with a doubling measure $\mu$ under some mild regularity conditions on the measure $\mu$. We also give sufficient conditions on a bounded domain to support fractional Poincaré type inequalities in this setting.
\end{abstract}

\section{Introduction}

The aim of this paper is to study improved fractional Poincaré type inequalities in a bounded John domain $\Omega \subset X$, where $(X, d, \mu)$ is a metric space endowed with a doubling measure $\mu$.

Recall that, roughly speaking, $\Omega$ is a John domain if it has a central point such that any other point can be connected to it without getting too close to the boundary (see Section 2 for a precise definition), and that this is essentially the largest class of domains in $\mathbb{R}^{n}$ for which the Sobolev-Poincaré inequality with Lebesgue measure,

$$
\left\|u-u_{\Omega}\right\|_{L^{\frac{n p}{n-p}}(\Omega)} \leq C\left(\int_{\Omega}|\nabla u(x)|^{p} d x\right)^{\frac{1}{p}},
$$

holds (see [3], [18], [39], [43] for the sufficiency, and [5] for the necessity). Here, $u$ is a locally Lipschitz function, $1 \leq p<n$ and $u_{\Omega}$ is the average of $u$ over $\Omega$.

The above inequality, also called $\left(\frac{n p}{n-p}, p\right)$-Poincaré inequality, is a special case of a larger family of so-called improved Poincaré inequalities, which are $(q, p)$-Poincaré inequalities with a weight that is a power of the distance to the boundary $d(x)$, namely,

$$
\left\|u-u_{\Omega}\right\|_{L^{q}(\Omega)} \leq C\left\|d^{\alpha}|\nabla u|\right\|_{L^{p}(\Omega)}
$$


where $1 \leq p \leq q \leq \frac{n p}{n-p(1-\alpha)}, p(1-\alpha)<n$ and $\alpha \in[0,1]$ (see [2], [23], and also [1], [11] for weighted versions).

A classical technique for getting this kind of inequalities is through the use of a representation formula in terms of a fractional integral, as can be seen for instance in [11], [24]. Another classical argument goes through the use of chains of cubes in order to reduce the problem of finding an inequality in $\Omega$ to its counterpart on these cubes. An approach which avoids the use of any representation formula to obtain Poincaré-Sobolev inequalities on cubes (or balls) was introduced in [15] (and then sharpened in [35]). See also the recent work [41] for more precise results on this direction. The local-to-global method began with the work [4] and later with [29], [31], and has been used by many authors, for example [7], [23] and [25], where both the integral representation formula and the local-to-global methods are used.

It is also worth noting that these inequalities have also been studied in metric spaces with doubling measures, replacing $|\nabla u|$ by a generalized gradient (see [20] and references therein).

In recent years, several authors have turned their attention to the fractional counterpart of inequality (1.1), beginning with the work [24] where the inequality

$$
\left\|u-u_{\Omega}\right\|_{L^{q}(\Omega)} \leq C\left(\int_{\Omega} \int_{\Omega \cap B(x, \tau d(x))} \frac{|u(x)-u(z)|^{p}}{|x-z|^{n+s p}} d x d z\right)^{1 / p}
$$

was obtained for a bounded John domain $\Omega, s, \tau \in(0,1), p<\frac{n}{s}$ and $1<p \leq q \leq \frac{n p}{n-s p}$. The case $p=1$ was proved in [13] using the so-called Maz'ya truncation method (see [39]) adapted to the fractional setting, which allows to obtain a strong inequality from a weak one.

Observe that the seminorm appearing on the right hand side of inequality (1.2) is weaker than that of the usual fractional Sobolev space $W^{s, p}(\Omega)$. More precisely, if we consider $W^{s, p}(\Omega)$ to be the subspace of $L^{p}(\Omega)$ induced by the seminorm

$$
[f]_{W^{s, p}(\Omega)}:=\left(\int_{\Omega} \int_{\Omega} \frac{|u(x)-u(z)|^{p}}{|x-z|^{n+s p}} d x d z\right)^{1 / p}
$$

and $\widetilde{W}^{s, p}(\Omega)$ to be the one induced by the seminorm

$$
[f]_{\widetilde{W}^{s, p}(\Omega)}:=\left(\int_{\Omega} \int_{\Omega \cap B(z, \tau d(z))} \frac{|u(x)-u(z)|^{p}}{|x-z|^{n+s p}} d x d z\right)^{1 / p},
$$

for fixed $s, \tau \in(0,1)$, then it is known that both spaces coincide when $\Omega$ is Lipschitz (see [12]), but there are examples of John domains $\Omega \subset \mathbb{R}^{n}$ for which the inclusion $W^{s, p}(\Omega) \subset \widetilde{W}^{s, p}(\Omega)$ is strict (see [9] for this result and characterizations of both 
spaces as interpolation spaces). This has led to call inequality (1.2) an "improved" fractional inequality. However, throughout this work, we will use this terminology to refer to inequalities including powers of the distance to the boundary as weights, as in the classical case.

Improvements of inequality (1.2) in this sense were obtained in [10] by including powers of the distance to the boundary to appropriate powers as weights on both sides of the inequality, and in [34], where the weights are defined by powers of the distance to a compact set of the boundary of the domain.

In this article we deal with the natural problem of extending the fractional inequalities mentioned above to metric measure spaces. To the best of our knowledge the results about fractional Poincaré inequalities in this setting are new. We will consider a metric measure space $(X, d, \mu)$, where $\mu$ is a Borel doubling measure satisfying some mild regularity assumptions. To this end, for a given $\Omega \subset X, 1 \leq p<\infty$, $\tau, s \in(0,1)$, and a weight $w$ (i.e., a locally integrable $\mu$-almost everywhere positive function), we define the seminorm

$$
[u]_{W_{\tau}^{s, p}(\Omega, w)}:=\left(\int_{\Omega} \int_{\Omega \cap\{d(z, y) \leq \tau d(y)\}} \frac{|u(z)-u(y)|^{p} w(z, y) d \mu(z) d \mu(y)}{\mu[B(z, d(z, y))] d(z, y)^{s p}}\right)^{1 / p} .
$$

It should be pointed out that this is the natural extension of the fractional Sobolev space to the context of metric spaces. Indeed, according to [17] the Besov space $B_{p, q}^{s}(X, d \mu)$ defined by the norm

$$
\left(\int_{X} \int_{X} \frac{|u(x)-u(y)|^{p}}{\mu[B(x, d(x, y))] d(x, y)^{s p}} d \mu(x) d \mu(y)\right)^{1 / p}
$$

coincides with the interpolation space between $L^{p}(X, d \mu)$ and the Sobolev space $W^{1, p}(X, d \mu)$ in the case that the metric supports a Poincaré inequality. More details about this fact and Besov spaces can be found in [17], [19], [20], [42], [47].

In this work, we are interested in the study of inequalities of the form

$$
\inf _{a \in \mathbb{R}}\|u-a\|_{L^{q}(\Omega, w d \mu)} \lesssim[u]_{W_{\tau}^{s, p}(\Omega, v d \mu)}
$$

where $1 \leq p, q<\infty, s, \tau \in(0,1)$ and $w, v$ are weights. We will say that $\Omega$ supports the $(w, v)$-weighted fractional ( $q, p)$-Poincaré inequality in $\Omega$ if (1.3) holds on $\Omega$ for every function $u \in L^{p}(\Omega, w d \mu)$ for which the right hand side is finite. When $w$ and $v$ are defined by functions of the distance to the boundary, we shall refer to these inequalities as $(w, v)$-improved fractional inequalities.

Our first goal is to obtain such inequalities with weights of the form $w_{\phi}^{F}(x)=$ $\phi\left(d_{F}(x)\right)$, where $\phi$ is a positive increasing function satisfying a certain growth condition and $F$ is a compact set in $\partial \Omega$. The parameter $F$ in the notation will be 
omitted whenever $F=\partial \Omega$. At the right hand side of the inequality, we will obtain a weight of the form $v_{\Phi, \gamma}^{F}(x, y)=\min _{z \in\{x, y\}} d(z)^{\gamma} \Phi\left(d_{F}(z)\right)$, where $\Phi$ will be an appropriate power of $\phi$. Our results extend and improve results in [10], [24], [34] in several ways. On one hand, the obtained class of weights is larger than the ones previously considered, even in the Euclidean setting. On the other hand, our inequalities hold for a very general class of spaces. Among these we can find, of course, the usual Euclidean space, but other important examples are included (see the discussion in Section 4).

Our second goal is to prove a sufficient condition for a domain $\Omega$ and a function $\phi$ to support an improved $(q, p)$-Poincaré inequality considering weights $w_{\phi}$ of the same type as the ones obtained above. In the Euclidean case it is well-known that if $q<p$, inequality (1.2) does not hold for general domains. Indeed, it was shown in [24, Theorem 6.9] that there exists a $\delta$-John domain which does not support this inequality. Following the ideas in [24] we obtain geometric sufficient conditions on a bounded domain $\Omega \subset X$ and a function $\phi$ to support an improved fractional $(q, p)$-Poincaré-Sobolev inequality when $q \leq p$ in the setting of metric measure spaces.

The rest of the paper is organized as follows: in Section 2 we introduce some necessary notations and previous results; Section 3 is devoted to prove our $(w, v)$-improved fractional $(q, p)$-Poincaré inequalities for $1 \leq p \leq q<\infty$. In Section 4 we consider some special cases of those inequalities. In Section 5 we give the proof of the sufficient condition for a bounded domain to support the $(q, p)$-Poincaré inequality for $q \leq p$. Finally, in Section 6 we give an example of a domain satisfying the condition of Theorem 4.

\section{Notations and preliminaries}

From now on $C$ and $c$ will denote constants that can change their values even within a single string of estimates. When necessary, we will stress the dependence of a constant on a particular parameter by writing it as a subindex. Also, we will use the notation $A \lesssim B$ whenever there exists a constant $c>0$ independent of all relevant parameters for which $A \leq c B$. Whenever $A \lesssim B$ and $B \lesssim A$, we will write $A \asymp B$.

A metric space $(X, d)$ is a set $X$ with a metric $d$, namely a nonnegative function defined on $X \times X$ such that

(1) For every $(x, y) \in X \times X, d(x, y)=0$ if and only if $x=y$.

(2) For every $(x, y) \in X \times X, d(x, y)=d(y, x)$.

(3) The inequality $d(x, y) \leq d(x, z)+d(y, z)$ holds for every $x, y, z \in X$.

The distance between a point $x$ and a subset $F$ of the boundary of $\Omega$ will be denoted $d_{F}(x):=d(x, F)$. When $F=\partial \Omega$, we will simply write $d(x):=d(x, \partial \Omega)$. For 
given $r>0$ and $x \in X$, the ball centered at $x$ with radius $r$ is the set $B(x, r):=\{y \in$ $X: d(x, y)<r\}$. Given a ball $B \subset X, r(B)$ will denote its radius and $x_{B}$ its center. For any $\lambda>0, \lambda B$ will be the ball with same center as $B$ and radius $\lambda r(B)$.

A doubling metric space is a metric space $(X, d)$ with the following (geometric) doubling property: there exists a positive integer $N \in \mathbb{N}$ such that, for every $x \in$ $X$ and $r>0$, the ball $B(x, r)$ can be covered by at most $N$ balls $B\left(x_{i}, r / 2\right)$ with $x_{1}, \ldots, x_{N} \in X$.

Every doubling metric space $(X, d)$ has a dyadic structure, as was proved by Hytönen and Kairema in the following theorem [26]:

Theorem A. Suppose that there are constants $0<c_{0} \leq C_{0}<\infty$ and $s \in(0,1)$ such that

$$
12 C_{0} s \leq c_{0}
$$

Given a set of points $\left\{z_{j}^{k}\right\}_{j}, j \in \mathbb{N}$, for every $k \in \mathbb{Z}$ - called dyadic points - with the properties that

$$
d\left(z_{i}^{k}, z_{j}^{k}\right) \geq c_{0} s^{k}, \quad i \neq j, \quad \min _{j \in \mathbb{N}} d\left(x, z_{j}^{k}\right)<c_{0} s^{k}, \quad x \in X,
$$

we can construct families of Borel sets $\widetilde{Q}_{j}^{k} \subset Q_{j}^{k} \subset \bar{Q}_{j}^{k}-$ called open, half open and closed dyadic cubes - such that:

$$
\begin{aligned}
& \widetilde{Q}_{j}^{k} \text { and } \bar{Q}_{j}^{k} \text { are interior and closure of } Q_{j}^{k}, \text { respectively; } \\
& \text { if } l \geq k, \text { then either } Q_{i}^{l} \subset Q_{j}^{k} \text { or } Q_{j}^{k} \cap Q_{i}^{l}=\varnothing ; \\
& X=\bigcup_{j \in \mathbb{N}} Q_{j}^{k}, \quad k \in \mathbb{Z} ; \\
& b\left(Q_{j}^{k}\right):=B\left(z_{j}^{k}, c_{1} s^{k}\right) \subset \widetilde{Q}_{j}^{k} \subset Q_{j}^{k} \subset \bar{Q}_{j}^{k} \subset B\left(z_{j}^{k}, C_{1} s^{k}\right)=: B\left(Q_{j}^{k}\right),
\end{aligned}
$$

where $c_{1}:=\frac{c_{0}}{3}$ and $C_{1}:=2 C_{0}$;

$$
\text { if } k \leq l \text { and } Q_{i}^{l} \subset Q_{j}^{k} \text {, then } B\left(Q_{i}^{l}\right) \subset B\left(Q_{j}^{k}\right) \text {. }
$$

Remark 1.

(1) One can always find a family of points $\left\{z_{j}^{k}\right\}_{j \in \mathbb{N}, k \in \mathbb{Z}}$ as the one in the hypothesis, so a doubling metric space always has a dyadic structure.

(2) The open and closed cubes are indeed open and closed sets, respectively.

The metric space $(X, d)$ will be endowed with a (always nonzero) Borel measure $\mu$ and denoted by $(X, d, \mu)$. If $E$ is a measurable set and $u$ is a measurable function, we write $u_{E}=\frac{1}{\mu(E)} \int_{E} u d \mu$ for the average of $u$ over $E$. We say that $\mu$ is doubling if for any ball $B \subset X$ there exists a constant $C_{d}$ depending on the measure such that 
$\mu(2 B) \leq C_{d} \mu(B)$. Equivalently, $\mu$ is a doubling (or $n_{\mu}$-doubling) measure if there exist constants $c_{d}, n_{\mu}>0$ such that

$$
\frac{\mu(\widetilde{B})}{\mu(B)} \leq c_{d}\left(\frac{r(\widetilde{B})}{r(B)}\right)^{n_{\mu}},
$$

for any pair of balls $B \subset \widetilde{B}$ in $X$. It turns out that any metric space endowed with a doubling measure is a doubling metric space. Observe that, if $0 \leq n_{\mu} \leq \eta$, then (2.7) also holds for $\eta$ instead of $n_{\mu}$.

We will also need to consider another property for the doubling measure $\mu$. Take $\delta>0$. We say that a measure $\mu$ is a $\delta$-reverse doubling measure, if there exists $c_{r}>0$ such that

$$
c_{\delta}\left(\frac{r(\widetilde{B})}{r(B)}\right)^{\delta} \leq \frac{\mu(\widetilde{B})}{\mu(B)},
$$

for any pair of balls $B \subset \widetilde{B}$ in $X$. Observe that, if $0 \leq s \leq \delta$, then (2.8) also holds for $s$ instead of $\delta$.

It should be noted that, for an $n_{\mu}$-doubling and $\delta$-reverse doubling measure $\mu$, the relation $\delta \leq n_{\mu}$ must be satisfied. When we say that $\mu$ is $\delta$-reverse doubling, we will always assume that $\delta$ is the biggest exponent for which there exists $c_{\delta}>0$ such that (2.8) holds. Analogously, $n_{\mu}$ will be assumed to be the smallest exponent for which there exists $c_{d}$ such that (2.7) holds.

The reverse doubling property is not too restrictive, as doubling measures are reverse doubling whenever the metric space satisfies some metric property called uniform perfectness (see, e.g., [40], [46]). Also, it is known (see [21, p. 112]) that Ahlfors-David regular spaces are precisely (up to some transformations) those uniformly perfect metric spaces carrying a doubling measure.

Recall that, for $\eta>0$, a measure $\mu$ is $\eta$-Ahlfors-David regular if there exist constants $c_{l}, c_{u}>0$ such that

$$
c_{l} r(B)^{\eta} \leq \mu(B) \leq c_{u} r(B)^{\eta},
$$

for any ball $B$ with $x_{B} \in X, 0<r(B)<\operatorname{diam} X$. The measure $\mu$ will be called $\eta$-lower Ahlfors-David regular if it satisfies just the left-hand side inequality and will be called $\eta$-upper Ahlfors-David regular if it satisfies just the right-hand side one.

For a subset $E \subset X$, we will say that $\mu$ is (resp. lower or upper) Ahlfors-David regular on $E$ if the induced subspace $\left(E,\left.d\right|_{E},\left.\mu\right|_{E}\right)$ is (resp. lower or upper) AhlforsDavid regular.

Along this paper, $\Omega$ will be a domain, i.e. an open connected set. The wellknown Whitney decomposition in the Euclidean case can be extended to a doubling metric space $(X, d)$, see for instance [8], [22], [36]. 
Lemma B. (Whitney decomposition [22]) Let $\Omega$ be domain of finite measure strictly contained in $X$. For fixed $M>5$, we can build a covering $W_{M}$ of $\Omega$ by a countable family of balls $\left\{B_{i}\right\}_{i \in \mathbb{N}}$ such that $B_{i}=B\left(x_{i}, r_{i}\right)$, with $x_{i} \in \Omega$ and $r_{i}=r\left(B_{i}\right)=$ $\frac{1}{M} d\left(x_{i}\right), i \in \mathbb{N}$.

Remark 1. The Whitney decomposition can be taken such that, if one denotes $B^{*}$ the dilation of a ball $B$, then, when the chosen dilation is sufficiently small compared to $M$, we get the following properties for any ball $B \in W_{M}$ and their dilations:

(1) $B^{*} \subset \Omega$;

(2) $c_{M}^{-1} r\left(B^{*}\right) \leq d(x) \leq c_{M} r\left(B^{*}\right)$, for all $x \in B^{*}$;

(3) $\sum_{B \in W_{M}} \chi_{B^{*}} \lesssim \chi_{\Omega}$.

We note that, for a fixed ball $B_{0} \in W_{M}$ and any ball $B$ in $W_{M}$, it is possible to build a finite chain $\mathcal{C}\left(B^{*}\right):=\left(B_{0}^{*}, B_{1}^{*}, \ldots, B_{k}^{*}\right)$, with $B_{k}=B$ in such a way that $\lambda B_{i} \cap \lambda B_{j} \neq \varnothing$ for some $\lambda>1$ sufficiently small (this $\lambda$ has something to do with the chosen dilation in the definition of $\left.B^{*}\right)$ if and only if $|i-j| \leq 1$. With this definition of $B^{*}$, we have that, for two consecutive balls $B_{j}^{*}$ and $B_{j+1}^{*}$ in a chain like this, the following property holds

$$
\max \left\{\mu\left(B_{j}^{*}\right), \mu\left(B_{j+1}^{*}\right)\right\} \leq c_{M, \lambda} \mu\left(B_{j}^{*} \cap B_{j+1}^{*}\right) .
$$

We denote $\ell\left[\mathcal{C}\left(B^{*}\right)\right]$ the length $k$ of this chain. Once the chains have been built, we can define, for each Whitney ball $E \in W_{M}$, the shadow of $E$ as the set $E\left(W_{M}\right)=\left\{B \in W_{M}: E^{*} \in \mathcal{C}\left(B^{*}\right)\right\}$. This construction is called a chain decomposition of $\Omega$ with respect to the fixed ball $B_{0}$.

The type of domains we consider in this work are the so-called John domains, first appeared in [32], and systematically studied since the work [37].

Definition 1. A domain $\Omega \subset X$ is a John domain if there is a distinguished point $x_{0} \in \Omega$ called central point and a positive constant $c_{J}$ such that every point $x \in \Omega$ can be joined to $x_{0}$ by a rectifiable curve $\gamma:[0, l] \rightarrow \Omega$ parametrized by its arc length for which $\gamma(0)=x, \gamma(l)=x_{0}$ and

$$
d(\gamma(t), \partial \Omega) \geq \frac{t}{c_{J}} \quad \text { for } t \in[0, l] .
$$

The following chaining result for John domains in doubling metric spaces is a slightly modified version of the chaining result in [20, Theorem 9.3]:

Theorem C. Let $\Omega$ be a John domain in a doubling metric space $(X, d)$. Let $C_{2} \geq 1$ and $x_{0}$ the central point of $\Omega$. Then, there exist a ball $B_{0}$ centered at $x_{0}$, and 
a constant $c_{2}$ that depends on the John constant of $\Omega$, the doubling constant of the space and $C_{2}$ such that for every $x \in \Omega$ there is a chain of balls $B_{i}=B\left(x_{i}, r_{i}\right) \subset \Omega$, $i=0,1, \ldots$, with the following properties

(1) There exists $R_{i} \subset B_{i} \cap B_{i+1}$ such that $B_{i} \cup B_{i+1} \subset c_{2} R_{i}, i=0,1, \ldots$;

(2) $d(x, y) \leq c_{2} r_{i}$, for any $y \in B_{i}, i=0,1, \ldots$, with $r_{i} \rightarrow 0$ for $i \rightarrow \infty$;

(3) $d\left(B_{i}, \partial \Omega\right) \geq C_{2} r_{i}, i=0,1, \ldots$;

(4) $\sum_{j} \chi_{B_{j}} \leq c_{2} \chi_{\Omega}$.

Remark 2. The sequence of balls obtained in [20, Theorem 9.3] is finite, but it can be easily completed to a (possibly) infinite one with the properties mentioned above (see also [24, Lemma 4.9] for the case of $\mathbb{R}^{n}$ ).

Remark 3. Theorem $\mathrm{C}$ holds for a larger class of domains called weak John domains, i.e., those domains $\Omega$ for which there are a central point $x_{0}$ and a constant $c_{J} \geq 1$ such that for every $x \in \Omega$ there exists a curve $\gamma:[0,1] \rightarrow \Omega$ with $\gamma(0)=x, \gamma(1)=$ $x_{0}$ and

$$
d\left(\gamma(t), \Omega^{c}\right) \geq \frac{d(x, \gamma(t))}{c_{J}}, \quad t \in[0,1]
$$

In fact, [20, Theorem 9.3] (and our modified version) is proved for bounded weak John domains. However, in this paper we will restrict ourselves to usual John domains.

We will also need the following result about the boundedness of certain type of operators from $L^{p}(X, \mu)$ to $L^{q}(X, \mu)$. This result can be found in a more general version in [44, Theorem 3].

Theorem D. Let $(X, d, \mu)$ be a metric space endowed with a doubling measure $\mu$. Set $1<p<q<\infty$. Let $T$ be an operator given by

$$
T f(x)=\int_{X} K(x, y) f(y) d \mu(y), \quad x \in X
$$

where $K(x, y)$ is a nonnegative kernel.

Let us define

$$
\varphi(B)=\sup \{K(x, y): x, y \in B, d(x, y) \geq C(K) r(B)\}
$$

where $B$ is a ball of radius $r(B)$ and $C(K)$ is a sufficiently small positive constant that depends only on the metric $d$ and the kernel $K$. 
Suppose that

$$
\sup _{B \subset X} \varphi(B) \mu(B)^{\frac{1}{q}+\frac{1}{p^{\prime}}}<\infty,
$$

where the supremum is taken over all the balls $B \subset X$.

Under these hypotheses,

$$
\left(\int_{X}|T f(x)|^{q} d \mu(x)\right)^{1 / q} \lesssim\left(\int_{X} f(x)^{p} d \mu(x)\right)^{1 / p} .
$$

This result can be used to bound the fractional integral operator in our context under mild conditions on the measure. Recall that

$$
I_{\alpha}^{\mu} f(x):=\int_{X} \frac{f(y) d(x, y)^{\alpha}}{\mu[B(x, d(x, y))]} d \mu(y), \quad 0<\alpha .
$$

By $I_{0}^{\mu}$ we understand the Hardy-Littlewood maximal function associated to the measure $\mu$, namely

$$
I_{0}^{\mu} f(x)=M_{\mu} f(x):=\sup _{B \ni x} \frac{1}{\mu(B)} \int_{B}|f(x)| d \mu(x) .
$$

When $\alpha=0$ (i.e., for the maximal function), the only possibility is $p=q$ and, in this case, it is enough to ask the measure $\mu$ to be doubling. Let then $\alpha>0$ and $p>1$. For some $q>p$ to be chosen later, we shall bound this operator from $L_{\mu}^{q^{\prime}}$ to $L_{\mu}^{p^{\prime}}$ using Theorem D.

To check that (2.12) holds, fix a ball $B$. Then, by the doubling condition, for any $x, y \in B$ with $d(x, y) \geq C_{\mu, \alpha} r(B)$ we have that

$$
\frac{d(x, y)^{\alpha}}{\mu[B(x, d(x, y))]} \mu(B)^{1 / p^{\prime}+1 / q} \asymp \frac{r(B)^{\alpha}}{\mu(B)} \mu(B)^{1 / p^{\prime}+1 / q},
$$

and then a sufficient condition for the boundedness of our operator is

$$
\sup _{B \subset X} r(B)^{\alpha} \mu(B)^{1 / q-1 / p}<\infty .
$$

This tells us that if our measure $\mu$ is $\alpha \frac{p q}{q-p}$-lower Ahlfors-David regular, then the claimed boundedness holds.

Observe that, as can be deduced from [44, Theorem 3], if the measure is $\alpha$-reverse doubling, then the $\alpha \frac{p q}{q-p}$-lower Ahlfors-David regularity is a necessary and sufficient condition for the boundedness of the operator.

If we let $\eta:=\alpha \frac{p q}{q-p}$, then we may write $q$ in the form $q=\frac{\eta p}{\eta-\alpha p}$. It is immediate that measures that are Ahlfors-David regular on the whole space are automatically doubling and reverse doubling. 


\section{Fractional Poincaré-Sobolev inequalities on John domains}

This section is devoted to the study of improved fractional $(q, p)$-Poincaré inequalities on bounded John domains, where $1 \leq p \leq q<\infty$. Particular cases of these inequalities include some already known results in the Euclidean case, such as the unweighted inequalities considered in [24] and the inequalities where the weights are powers of the distance to the boundary considered in [10], [34]. We will come back to these special examples in Section 4.

The proof makes use of some of the arguments in [24] and [10]. The fundamental idea is the classical one to obtain ordinary Poincaré inequalities: to bound the oscillation of the function $u$ by the fractional integral of its derivative by using regularity properties of the function and the domain and then use the boundedness properties of the fractional integral operator. Thus, if we understand the function

$$
g_{p}(y):=\left[\int_{\{z \in \Omega: d(z, y) \leq \tau d(y)\}} \frac{|u(y)-u(z)|^{p}}{\mu[B(z, d(y, z))] d(y, z)^{s p}} d \mu(z)\right]^{1 / p} \chi_{\Omega}(y), \quad y \in \Omega
$$

as a fractional version of the derivative of $u$ on $\Omega$, we just have to bound $|u(x)-a|$ for some $a \in \mathbb{R}$ by the fractional integral $I_{s}^{\mu} g_{p}(x)$, for $\mu$-a.e. $x \in \Omega$ (see (2.14) for the definition of the fractional integral). This is done in the following lemma.

Lemma 1. Consider a bounded John domain $\Omega$ in the doubling metric space $(X, d, \mu)$ with a chain as the one in Theorem $C$. Suppose $\mu$ to be $\delta$-reverse doubling. Let $s, \tau \in(0,1), 0 \leq s \leq \delta$ and $1 \leq p<\infty$. There exists $c_{3}>0$ such that, for any $u \in$ $W^{s, p}(\Omega, \mu)$.

$$
\left|u(x)-u_{B_{0}}\right| \lesssim I_{s}^{\mu}\left(g_{p} \chi_{\Omega \cap\left\{d(\cdot, x) \leq c_{3} d(\cdot)\right\}}\right), \quad \mu-\text { a.e. } x \in \Omega,
$$

where $g_{p}$ is as in (3.1).

Proof. We can use the chain of balls in Theorem $\mathrm{C}$ and the Lebesgue differentiation theorem in order to obtain, for $\mu$-almost every $x \in \Omega$,

$$
u(x)=\lim _{i \rightarrow \infty} \frac{1}{\mu\left(B_{i}\right)} \int_{B_{i}} u(y) d \mu(y)=\lim _{i \rightarrow \infty} u_{B_{i}} .
$$

Fix one of these points $x \in \Omega$. Then, as consecutive balls in the chain intersect in a ball whose dilation contain the union of them, we have

$$
\begin{aligned}
\left|u(x)-u_{B_{0}}\right| & \leq \sum_{i=0}^{\infty}\left|u_{B_{i+1}}-u_{B_{i}}\right| \\
& \leq \sum_{i=0}^{\infty}\left|u_{B_{i+1}}-u_{B_{i} \cap B_{i+1}}\right|+\left|u_{B_{i}}-u_{B_{i} \cap B_{i+1}}\right| \\
& \lesssim \sum_{i=0}^{\infty} \frac{1}{\mu\left(B_{i}\right)} \int_{B_{i}}\left|u(y)-u_{B_{i}}\right| d \mu(y) .
\end{aligned}
$$


Now, observe that, for $z, y \in B_{i}$ we have that $B(y, d(z, y)) \subset 3 B_{i}$. Then, we have that each term in the sum above can be bounded as follows

$$
\begin{aligned}
\frac{1}{\mu\left(B_{i}\right)} \int_{B_{i}}\left|u(y)-u_{B_{i}}\right| d \mu(y) & \leq \frac{1}{\mu\left(B_{i}\right)} \int_{B_{i}}\left|\frac{1}{\mu\left(B_{i}\right)} \int_{B_{i}}(u(y)-u(z)) d \mu(z)\right| d \mu(y) \\
& \lesssim \frac{r_{i}^{s}}{\mu\left(B_{i}\right)} \int_{B_{i}}\left(\int_{B_{i}} \frac{|u(y)-u(z)|^{p} d \mu(z)}{\mu\left(B_{i}\right) d(y, z)^{s p}}\right)^{1 / p} d \mu(y) \\
& \lesssim \frac{r_{i}^{s}}{\mu\left(B_{i}\right)} \int_{B_{i}}\left(\int_{B_{i}} \frac{|u(y)-u(z)|^{p} d \mu(z)}{\mu[B(y, d(y, z))] d(y, z)^{s p}}\right)^{1 / p} d \mu(y) .
\end{aligned}
$$

According to condition (3) from Theorem $\mathrm{C}$ we have $d\left(B_{i}, \partial \Omega\right) \geq C_{2} r_{i}$ for every $i=0,1, \ldots$, so

$$
d(y) \geq C_{2} r_{i}, \quad y \in B_{i},
$$

and thus, for any $y, z \in B_{i}$ we can write $d(y, z) \leq 2 r_{i} \leq \frac{2}{C_{2}} d(y)$. Hence, by choosing $C_{2}=\frac{2}{\tau}$

$$
\begin{aligned}
& \frac{1}{\mu\left(B_{i}\right)} \int_{B_{i}}\left|u(y)-u_{B_{i}}\right| d \mu(y) \\
& \quad \lesssim \frac{r_{i}^{s}}{\mu\left(B_{i}\right)} \int_{B_{i}}\left(\int_{\{z \in \Omega: d(y, z) \leq \tau d(y)\}} \frac{|u(y)-u(z)|^{p} d \mu(z)}{\mu[B(y, d(y, z))] d(y, z)^{s p}}\right)^{1 / p} d \mu(y) \\
& \quad=\frac{r_{i}^{s}}{\mu\left(B_{i}\right)} \int_{B_{i}} g_{p}(y) d \mu(y) .
\end{aligned}
$$

Therefore

$$
\sum_{i=0}^{\infty} \frac{1}{\mu\left(B_{i}\right)} \int_{B_{i}}\left|u(y)-u_{B_{i}}\right| d \mu(y) \lesssim \sum_{i=0}^{\infty} \frac{r_{i}^{s}}{\mu\left(B_{i}\right)} \int_{B_{i}} g_{p}(y) d \mu(y) .
$$

Now, by (2) in Theorem C, the doubling and $\delta$ - reverse doubling property of $\mu$, we have that

$$
\frac{r_{i}^{s}}{\mu\left(B_{i}\right)} \int_{B_{i}} g_{p}(y) d \mu(y) \lesssim \int_{B_{i}} \frac{g_{p}(y) d(x, y)^{s}}{\mu[B(x, d(x, y))]} d \mu(y), \quad i=0,1, \ldots
$$

and from this and the fact that $d(x, y) \leq \frac{c_{2}}{C_{2}} d(y)$ for every $y \in B_{i}$, we deduce

$$
\sum_{i=1}^{\infty} \frac{1}{\mu\left(B_{i}\right)} \int_{B_{i}}\left|u(y)-u_{B_{i}}\right| d \mu(y) \lesssim \int_{\Omega \cap\left\{d(x, y) \leq c_{3} d(y)\right\}} \frac{g_{p}(y) d(x, y)^{s}}{\mu[B(x, d(x, y))]} d \mu(y),
$$

where $c_{3}:=\frac{c_{2}}{C_{2}}=\frac{\tau c_{2}}{2}$. 
We will also need the following lemma for our main theorem.

Lemma 2. Let $s>0$. Then, for any $x \in X$ and any $\varepsilon>0$ we have

$$
I_{s}^{\mu}\left(f \chi_{\Omega \cap\{d(x, \cdot)<\varepsilon\}}\right)(x) \lesssim \varepsilon^{s} I_{0}^{\mu}\left(f \chi_{\Omega}\right)(x)
$$

Proof. A standard argument (see [28]) dividing the integral gives us, for any $x \in X$,

$$
\begin{aligned}
I_{s}^{\mu} & \left(f \chi_{\Omega \cap\{d(x, \cdot)<\varepsilon\}}\right)(x) \\
& =\int_{d(x, y)<\varepsilon} \frac{f(y) \chi_{\Omega}(y)}{\mu[B(x, d(x, y))] d(x, y)^{-s}} d \mu(y) \\
& =\sum_{k=0}^{\infty} \int_{B\left(x, \frac{\varepsilon}{2^{k}}\right) \backslash B\left(x, \frac{\varepsilon}{2^{k+1}}\right)} \frac{f(y) \chi_{\Omega}(y)}{\mu[B(x, d(x, y))] d(x, y)^{-s}} d \mu(y) \\
& \lesssim \varepsilon^{s} \sum_{k=0}^{\infty} \frac{1}{\mu\left[B\left(x, \frac{\varepsilon}{2^{k}}\right)\right] 2^{s(k+1)}} \int_{B\left(x, \frac{\varepsilon}{2^{k}}\right) \backslash B\left(x, \frac{\varepsilon}{2^{k+1}}\right)} f(y) \chi_{\Omega}(y) d \mu(y) \\
& \lesssim \varepsilon^{s} I_{0}^{\mu}\left(f \chi_{\Omega}\right)(x) . \quad \square
\end{aligned}
$$

Now we are ready to prove the main results of the section. Recall that we will work with weights of the form $w_{\phi}^{F}(x)=\phi\left(d_{F}(x)\right)$, where $F$ is omitted whenever $F=$ $\partial \Omega$, and that $\phi$ is a positive increasing function that satisfies the growth condition $\phi(2 x) \leq C \phi(x)$ for all $x \in \mathbb{R}_{+}$. Observe that this implies $\phi(k x) \leq C_{k} \phi(x)$ for every $k \geq 1$. We will obtain, at the right hand side of the inequality, a weight of the form $v_{\Phi, \gamma p}^{F}(x, y)=\min _{z \in\{x, y\}} d(z)^{\gamma p} \Phi\left(d_{F}(z)\right)$, where $\Phi$ is an appropriate power of $\phi$.

Theorem 1. Let $(X, d, \mu)$ be a metric space with $n_{\mu}$-Ahlfors-David regularity. Let $s, \tau \in(0,1)$ and $0 \leq \gamma<s \leq n_{\mu}$. Let $1<p<\infty$ be such that $(s-\gamma) p<n_{\mu}$ and take $p_{s-\gamma}^{*}:=\frac{n_{\mu} p}{n_{\mu}-(s-\gamma) p}$. Let $\Omega \subset X$ be a bounded John domain. Let $F \subset \partial \Omega$ be a compact set. Consider a positive increasing function $\phi$ satisfying the growth condition $\phi(2 x) \leq C \phi(x)$ and such that $w_{\phi}^{F} \in L_{\mathrm{loc}}^{1}(\Omega, d \mu)$, and define the function $\Phi(t)=\phi(t)^{p / p_{s-\gamma}^{*}}$. Then, for any function $u \in W^{s, p}(\Omega, \mu)$,

$$
\inf _{a \in \mathbb{R}}\|u-a\|_{\left.L^{p_{s-\gamma}^{*}\left(\Omega, w_{\phi}^{F}\right.} d \mu\right)} \lesssim[u]_{W_{\tau}^{s, p}\left(\Omega, v_{\Phi, \gamma p}^{F} d \mu\right)} .
$$

Theorem 2. Let $(X, d, \mu)$ be a metric space with $\mu$ a doubling measure satisfying a $\delta$-reverse doubling property. Consider $w_{\phi}^{F}, F$ and $\phi$ as in the statement of Theorem 1. For $1 \leq p<\infty$ and $0<s \leq \delta$ we have the following $(p, p)$ Poincaré inequality

$$
\inf _{a \in \mathbb{R}}\|u-a\|_{L^{p}\left(\Omega, w_{\phi}^{F} d \mu\right)} \lesssim[u]_{W_{\tau}^{s, p}\left(\Omega, v_{\Phi, s p}^{F} d \mu\right)}
$$


In the sequel we will give only the proof of Theorem 1 given that the proof of Theorem 2 follows in the same way, recalling that $I_{0}^{\mu} f$ stands for the HardyLittlewood maximal operator. The $(1,1)$-inequality follows with this proof from the boundedness of $I_{0}^{\mu}$ in $L^{\infty}$.

Proof of Theorem 1. We proceed by duality. Let $f$ be such that $\|f\|_{L^{\left(p_{s-\gamma}^{*}\right)^{\prime}}\left(\Omega, w_{\phi}^{F} d \mu\right)}=1$. Then, by Lemma 1 and Tonelli's theorem, we have

$$
\begin{aligned}
& \int_{\Omega}\left|u(x)-u_{B_{0}}\right| f(x) w_{\phi}^{F}(x) d \mu(x) \\
& \lesssim \int_{\Omega} I_{s}^{\mu}\left(g_{p} \chi_{\left.\Omega \cap\left\{d(\cdot, x) \leq c_{3} d(\cdot)\right\}\right)(x) f(x) w_{\phi}^{F}(x) d \mu(x)}\right. \\
& =\int_{\Omega} \int_{\left\{x \in \Omega: d(y, x) \leq c_{3} d(y)\right\}} \frac{f(x)\left[w_{\phi}^{F}(x)\right]^{\frac{1}{\left(p_{s-\gamma}^{*}\right.}+\frac{1}{p_{s-\gamma}^{*}} d(x, y)^{s-\gamma+\gamma}}}{\mu[B(x, d(x, y))]} d \mu(x) \\
& \quad \times g_{p}(y) d \mu(y) .
\end{aligned}
$$

Now observe that, by hypothesis, $\phi\left(\left(1+c_{3}\right) t\right) \lesssim \phi(t)$. Hence, using Hölder's inequality and the boundedness properties of the operator (in the case of Theorem 2 we also use Lemma 2 and the boundedness of the Hardy-Littlewood maximal function) we may continue (3.2) with

$$
\begin{aligned}
& \int_{\Omega} I_{s-\gamma}^{\mu}\left[f\left[w_{\phi}^{F}\right]^{\frac{1}{\left(p_{s-\gamma}^{*}\right)^{\prime}}} \chi_{\left.\Omega \cap\left\{d(\cdot, y) \leq c_{3} d(y)\right\}\right]}(y) d(y)^{\gamma} \phi\left[d_{F}(y)\right]^{\frac{1}{p_{s-\gamma}^{*}}} g_{p}(y) d \mu(y)\right. \\
& \quad \lesssim\left(\int_{\Omega} \int_{\{x \in \Omega: d(x, y) \leq \tau d(y)\}} \frac{|u(x)-u(y)|^{p} d(y)^{\gamma p} \phi\left[d_{F}(y)\right]^{\frac{p}{p_{s}^{*}}}}{\mu[B(x, d(x, y))] d(x, y)^{s p}} d \mu(x) d \mu(y)\right)^{1 / p} \\
& \quad \lesssim\left(\int_{\Omega} \int_{\{x \in \Omega: d(x, y) \leq \tau d(y)\}} \frac{|u(x)-u(y)|^{p} v_{\Phi, \gamma p}^{F}(x, y)}{\mu[B(x, d(x, y))] d(x, y)^{s p}} d \mu(x) d \mu(y)\right)^{1 / p} \\
& \quad=[u]_{W_{\tau}^{s, p}\left(\Omega, v_{\Phi, \gamma p}^{F} d \mu\right)} \cdot \square
\end{aligned}
$$

Remark 4. It is possible to prove the same result whenever $(X, d, \mu)$ is a metric space with an $n_{\mu}$-doubling and $\delta$-reverse doubling measure $\mu$ with $\eta$-lower AhlforsDavid regularity for some $(s-\gamma) p<\eta$, where $0 \leq \gamma<s \leq \delta$ and $p>1$. In this case, the result is obtained with an $L^{p_{s-\gamma}^{*}}$ norm at the left-hand side, where $p_{s-\gamma}^{*}:=\frac{\eta p}{\eta-(s-\gamma) p}$. We remark that the $(p, p)$ (which corresponds to Theorem 2) does not need the lower Ahlfors-David regularity hypothesis as just the doubling property is needed for the boundedness of the Hardy-Littlewood maximal operator. Nevertheless, we decided to ask for more regularity in order to get cleaner statements. It should be 
noted that the growth condition on $\phi$ is not actually necessary, but the results are much cleaner assuming this condition.

In what follows, we prove the $\left(1_{s-\gamma}^{*}, 1\right)$-inequality by requiring some stronger properties on our measure $\mu$. For this, we will use the following lemma, which is a generalization of a well-known result which can be found, for instance, in the book by Jost [33].

Lemma 3. Take $0<\gamma<s, \eta>0$ and $q>1$. Let $(X, d, \mu)$ be a metric space with $\mu$ an $\eta$-upper and $(s-\gamma) q^{\prime}$-lower Ahlfors-David regular measure. Let $x \in X$ and suppose that for any measurable bounded set $F$ with positive measure there is a ball $B(x, R)$ with comparable measure to that of the set $F$. Then for any measurable set $E$ with positive measure we have that

$$
\int_{E} \frac{d \mu(y)}{d(x, y)^{(s-\gamma) q^{\prime}-(s-\gamma)}} \lesssim \mu(E)^{\frac{\eta+s-\gamma}{(s-\gamma) q^{\prime}}-1} .
$$

Proof. Let $R>0$ be such that the ball $B:=B(x, R)$ verifies $\mu(B) \asymp \mu(E)$. For this $R$, write

$$
\int_{E} \frac{d \mu(y)}{d(x, y)^{(s-\gamma) q^{\prime}-(s-\gamma)}}=\left(\int_{E \backslash(E \cap B)}+\int_{E \cap B}\right) \frac{d \mu(y)}{d(x, y)^{(s-\gamma) q^{\prime}-(s-\gamma)}} .
$$

On one hand, we note that for $y \in E \backslash(E \cap B)$, we have $d(x, y) \geq R$, so

$$
\int_{E \backslash(E \cap B)} \frac{d \mu(y)}{d(x, y)^{(s-\gamma) q^{\prime}-(s-\gamma)}} \leq \int_{E \backslash(E \cap B)} \frac{d \mu(y)}{R^{(s-\gamma) q^{\prime}-s}} \leq R^{\eta-(s-\gamma) q^{\prime}+s-\gamma},
$$

as $\mu$ is an $\eta$-upper Ahlfors-David regular measure.

On the other hand, for $y \in B$, we can use Lemma 2.1 in [16], so we obtain

$$
\int_{E \cap B} \frac{d \mu(y)}{d(x, y)^{(s-\gamma) q^{\prime}-(s-\gamma)}} \leq \int_{B} \frac{d \mu(y)}{d(x, y)^{(s-\gamma) q^{\prime}-(s-\gamma)}} \lesssim R^{\eta-(s-\gamma) q^{\prime}+s-\gamma} .
$$

Thus, as $\mu$ is $(s-\gamma) q^{\prime}$-lower Ahlfors-David regular and $\mu(B)=\mu(E)$, we finally get

$$
\int_{E} \frac{d \mu(y)}{d(x, y)^{(s-\gamma) q^{\prime}-(s-\gamma)}} \lesssim \mu(E)^{\frac{\eta+s-\gamma}{(s-\gamma) q^{\prime}}-1} .
$$

Remark 5. If $\mu$ is an $n_{\mu}$-Ahlfors-David regular measure, then the space $(X, d, \mu)$ satisfies that for any bounded measurable set $F$ there exists a ball of comparable size, and, hence, Lemma 3 holds. Indeed, for any $x \in X$ it suffices to take the ball $B\left(x, \frac{\mu(F)^{1 / n_{\mu}}}{2 c_{u}^{1 / n_{\mu}}}\right)$.

Theorem 3. Theorem 1 also holds for $p=1$. 
Proof. Let us define, for $\lambda>0$, the set $E:=\left\{x \in \Omega:\left|u(x)-u_{B_{0}}\right|>\lambda\right\}$ and assume that $\mu$ is $n_{\mu}$-lower and $n_{\mu}$-upper Ahlfors-David regular (note that $(s-\gamma)\left(1_{s-\gamma}^{*}\right)^{\prime}=$ $\left.(s-\gamma) \frac{n_{\mu}}{s-\gamma}=n_{\mu}\right)$. Then, by Chebyshev's inequality, Lemma 1 and Tonelli's theorem,

$$
\begin{aligned}
\left(w_{\phi}^{F} d \mu\right)(E) & \lesssim \frac{1}{\lambda} \int_{E} \int_{\Omega \cap\left\{d(x, y) \leq c_{3} d(y)\right\}} \frac{g_{1}(y) d(x, y)^{s}}{\mu[B(y, d(x, y))]} d \mu(y) w_{\phi}^{F}(x) d \mu(x) \\
& =\frac{1}{\lambda} \int_{\Omega} g_{1}(y) \int_{E \cap B\left(y, c_{3} d(y)\right)} \frac{w_{\phi}^{F}(x) d(x, y)^{s}}{\mu[B(y, d(x, y))]} d \mu(x) d \mu(y) \\
& =I_{1}+I_{2}
\end{aligned}
$$

where $I_{1}$ corresponds to the case in which the inner integral is defined on the region $E_{1}$ where $d(x, y) \leq \tau d(y)$ and $I_{2}$ to the case where the inner integral is evaluated on its complement, $E_{2}$. Observe that when $d(x, y) \leq \tau d(y)$, we have that $(1-\tau) d(y) \leq d(x) \leq(1+\tau) d(y)$ and that the same comparison holds for $d_{F}(x)$ and $d_{F}(y)$, so that, as $\mu$ is $n_{\mu}$-lower Ahlfors-David regular, by Lemma 3 and the fact that $\frac{n_{\mu}+s-\gamma}{(s-\gamma)\left(1_{s-\gamma}^{*}\right)^{\prime}}-1=\frac{1}{\left(1_{s-\gamma}^{*}\right)^{\prime}}$,

$$
\begin{aligned}
I_{1} & \lesssim \int_{\Omega} \frac{g_{1}(y)}{\lambda} \int_{E_{1}} \frac{d(x, y)^{s}}{\mu[B(y, d(x, y))]} d \mu(x) w_{\phi}^{F}(y) d \mu(y) \\
& \lesssim \int_{\Omega} d(y)^{\gamma} \frac{g_{1}(y)}{\lambda} \int_{E_{1}} \frac{d \mu(x)}{d(x, y)^{(s-\gamma)\left(1_{s-\gamma}^{*}\right)^{\prime}-(s-\gamma)}} w_{\phi}^{F}(y) d \mu(y) \\
& \lesssim \int_{\Omega} d(y)^{\gamma} \frac{g_{1}(y)}{\lambda} \mu\left(E_{1}\right)^{\frac{1}{\left(1_{s-\gamma}^{*}\right.}} w_{\phi}^{F}(y) d \mu(y) \\
& \lesssim \int_{\Omega} d(y)^{\gamma} \frac{g_{1}(y)}{\lambda}\left(\int_{E_{1}} w_{\phi}^{F}(x) d \mu(x)\right)^{\frac{1}{\left(1_{s-\gamma}^{*}\right)^{\prime}}}\left[w_{\phi}^{F}(y)\right]^{\frac{1}{1_{s-\gamma}^{*}}} d \mu(y) \\
& \lesssim \int_{\Omega} d(y)^{\gamma} \frac{g_{1}(y)}{\lambda}\left(w_{\phi}^{F} d \mu\right)(E)^{\frac{1}{\left(1_{s-\gamma}^{*}\right)^{\prime}}}\left[w_{\phi}^{F}(y)\right]^{\frac{1}{1_{s-\gamma}^{*}}} d \mu(y),
\end{aligned}
$$

where we have used that, by hypothesis, we know that $\phi\left((1+\tau) d_{F}(x)\right) \lesssim \phi\left(d_{F}(x)\right)$ and $\phi\left[\frac{(1+\tau)}{1-\tau} d_{F}(x)\right] \lesssim \phi\left(d_{F}(x)\right)$.

Hence, we have

$$
I_{1} \lesssim \int_{\Omega} \frac{g_{1}(y)}{\lambda}\left(w_{\phi}^{F} d \mu\right)(E)^{1 /\left(1_{s-\gamma}^{*}\right)^{\prime}}\left[w_{\phi}^{F}(y)\right]^{1 / 1_{s-\gamma}^{*}} d(y)^{\gamma} d \mu(y) .
$$


On the other hand, using that $d(x, y) \geq \tau d(y)$, we have that, as $\mu$ is $n_{\mu}$-upper Ahlfors-David regular and $s \leq n_{\mu}$,

$$
\begin{aligned}
I_{2} & =\frac{1}{\lambda} \int_{\Omega} g_{1}(y) \int_{E_{2}} \frac{w_{\phi}^{F}(x) d(x, y)^{s}}{\mu[B(y, d(x, y))]} d \mu(x) d \mu(y) \\
& \lesssim \frac{1}{\lambda} \int_{\Omega} g_{1}(y) d(y)^{s-n_{\mu}}\left(w_{\phi}^{F} d \mu\right)\left(E_{2}\right) d \mu(y) \\
& \lesssim \frac{1}{\lambda} \int_{\Omega} g_{1}(y) d(y)^{s-n_{\mu}}\left(w_{\phi}^{F} d \mu\right)\left(E_{2}\right)^{1 /\left(1_{s-\gamma}^{*}\right)^{\prime}} d(y)^{\frac{n_{\mu}}{1_{s-\gamma}^{*}}}\left[w_{\phi}^{F}(y)\right]^{1 / 1_{s-\gamma}^{*}} d \mu(y) \\
& \lesssim \frac{1}{\lambda} \int_{\Omega} g_{1}(y) d(y)^{\gamma}\left(w_{\phi}^{F} d \mu\right)(E)^{1 /\left(1_{s-\gamma}^{*}\right)^{\prime}}\left[w_{\phi}^{F}(y)\right]^{1 / 1_{s-\gamma}^{*}} d \mu(y) .
\end{aligned}
$$

Thus, we finally get

$$
\left(w_{\phi}^{F} d \mu\right)(E) \lesssim \frac{\left(w_{\phi}^{F} d \mu\right)(E)^{1 /\left(1_{s-\gamma}^{*}\right)^{\prime}}}{\lambda} \int_{\Omega} g_{1}(y) d(y)^{\gamma} w_{\phi}^{F}(y)^{1 / 1_{s-\gamma}^{*}} d \mu(y),
$$

i.e.

$$
\left\|u-u_{B_{0}}\right\|_{L^{1_{s-\gamma}^{*}, \infty}\left(\Omega, w_{\phi}^{F}\right)} \lesssim \int_{\Omega} g_{1}(y) d(y)^{\gamma} w_{\phi}^{F}(y)^{1 / 1_{s-\gamma}^{*}} d \mu(y) .
$$

At this point, a "weak implies strong" argument, which also holds in our setting (see the comments preceding [10, Lemma 3.2.] and also [14, Proposition 5], [18, Theorem 4], [13]) gives us the extremal case $p=1$ with weight $w_{\phi}^{F}$ at the left-hand side and $v_{\Phi, \gamma}^{F}$ at the right hand side.

Remark 6. In this case, Ahlfors-David regularity is needed for the argument, so Lemma 3 can be applied without any other assumption.

\section{Some particular cases of Theorems 1 and 2}

In this section we will give several instances of Theorems 1 and 2 by making specific choices of $(X, d, \mu)$ and $\phi$. These particular examples show that Theorems 1 and 2 extend results in [10], [24], [34] in several aspects.

Let us start with the Euclidean space with the Lebesgue measure, $\left(\mathbb{R}^{n}, d,|\cdot|\right)$, which is a doubling measure space with $n$-Ahlfors-David regularity. If we choose $\phi(t)=t^{a}$, where $a \geq 0, \Omega$ any bounded John domain in $\mathbb{R}^{n}$ and $F=\partial \Omega$, then we recover the results in [10] about John domains. More precisely,

Corollary 1. (Theorems 3.1. and 3.2. in [10]) Let $\Omega$ be a bounded John domain in $\mathbb{R}^{n}$. Let $\tau \in(0,1)$ and $a \geq 0$. Let $s \in(0,1)$ and take $1 \leq p<\infty$ such that 
$p s<n$. Thus, for any $q \leq p_{s}^{*}=\frac{p n}{n-s p}$, we have that, for any function $u \in W^{s, p}(\Omega, d x)$,

$$
\begin{aligned}
& \inf _{c \in \mathbb{R}}\|u-c\|_{L^{p_{s}^{*}}\left(\Omega, d^{a}\right)} \\
& \quad \lesssim\left(\int_{\Omega} \int_{\{z \in \Omega: d(z, y) \leq \tau d(y)\}} \frac{|u(z)-u(y)|^{p}}{|z-y|^{n+s p}} \delta(z, y)^{b} d z d y\right)^{\frac{1}{p}},
\end{aligned}
$$

where $\delta(z, y)=\min _{x \in\{z, y\}} d(x)$ and $b \leq a \frac{p}{q}+s p$.

Moreover, by choosing $F \varsubsetneqq \partial \Omega$ in Theorem 2 we recover the main result in [34], namely

Corollary 2. (Theorem 1.1 in [34]) Let $\Omega$ in $\mathbb{R}^{n}$ be a bounded John domain and $1<p<\infty$. Given $F$ a compact set in $\partial \Omega$, and the parameters $s, \tau \in(0,1)$ and $a \geq 0$, the inequality

$$
\begin{aligned}
& \inf _{c \in \mathbb{R}}\|u-c\|_{L^{p}\left(\Omega, d_{F}^{a}\right)} \\
& \quad \lesssim\left(\int_{\Omega} \int_{\{z \in \Omega: d(z, y) \leq \tau d(y)\}} \frac{|u(z)-u(y)|^{p}}{|z-y|^{n+s p}} \delta^{s p}(z, y) \delta_{F}(z, y)^{a} d z d y\right)^{\frac{1}{p}},
\end{aligned}
$$

holds for any function $u \in W^{s, p}(\Omega, d x)$, where $\delta_{F}(z, y)=\min _{x \in\{z, y\}} d_{F}(x)$.

If we use Theorem 1 for $F \varsubsetneqq \partial \Omega$, then we improve both results by obtaining the following combination of them:

Corollary 3. Let $\Omega$ be a bounded John domain in $\mathbb{R}^{n}$ and consider $F \subset \partial \Omega$. Let $\tau \in(0,1)$ and $a \geq 0$. Let $s \in(0,1)$ and take $1 \leq p<\infty$ such that $p s<n$. Thus, for any $q \leq p_{s}^{*}=\frac{p n}{n-s p}$, we have that, for any function $u \in W^{s, p}(\Omega, d x)$,

$$
\begin{aligned}
& \inf _{c \in \mathbb{R}}\|u-c\|_{L^{p_{s}^{*}}\left(\Omega, d_{F}^{a}\right)} \\
& \quad \lesssim\left(\int_{\Omega} \int_{\{z \in \Omega: d(z, y) \leq \tau d(y)\}} \frac{|u(z)-u(y)|^{p}}{|z-y|^{n+s p}} \delta(z, y)^{s p} \delta_{F}(z, y)^{b} d z d y\right)^{\frac{1}{p}},
\end{aligned}
$$

where $\delta_{F}(z, y)=\min _{x \in\{z, y\}} d_{F}(x)$ and $b \leq a \frac{p}{q}$.

In general, we are able to include in our inequalities a large class of weights defined by using the distance from the boundary. An instance of weights which is not included in the previous results is, for example, the family of weights $w_{\phi}^{F}$, where $\phi(t)=t^{a} \log ^{b}(e+t), a, b \geq 0$.

Another space on which our results can be applied is the Heisenberg group $\mathbb{H}^{n}$, as it is a $(2 n+2)$-Ahlfors-David regular metric measure space endowed with the Carnot-Carathéodory metric $d:=d_{C C}$ (see [45] for the definition of $d_{C C}$ ) and the Lebesgue measure. The result reads as follows:

Corollary 4. Let us consider the Heisenberg space $\left(\mathbb{H}^{n}, d_{C C}, \mathcal{H}^{2 n+2}\right)$, where $d_{C C}$ is the Carnot-Carathéodory metric and $\mathcal{H}^{2 n+2}$ is the $(2 n+2)$-dimensional Haus- 
dorff measure on $\mathbb{H}^{n}$. Let $\Omega$ be a bounded John domain in $\mathbb{H}^{n}$ and $F \subset \partial \Omega$ a compact set. Let $s, \tau \in(0,1)$ and $a \geq 0$ such that $d_{F}^{a} \in L_{\text {loc }}^{1}(\Omega)$. Let $0<\gamma \leq s$ and take $p>1$ such that $(s-\gamma) p<2 n+2$. Thus, for any $q \leq p_{s-\gamma}^{*}=\frac{p(2 n+2)}{(2 n+2)-(s-\gamma) p}$, we have that, for any function $u \in W^{s, p}\left(\Omega, \mathcal{H}^{2 n+2}\right)$,

$$
\begin{aligned}
& \left.\inf _{c \in \mathbb{R}}\|u-c\|_{L^{p_{s-\gamma}^{*}\left(\Omega, d_{F}^{a}\right.}}^{p} \mathcal{H}^{2 n+2}\right) \\
& \quad \lesssim \int_{\Omega} \int_{\Omega \cap B(y, \tau d(y))} \frac{|u(z)-u(y)|^{p} \delta_{F}^{\gamma p}(z, y) \delta(z, y)^{a \frac{p}{q}} d \mathcal{H}^{2 n+2}(z) d \mathcal{H}^{2 n+2}(y)}{\left(\left|z_{1}-y_{1}\right|^{4}+\left|z_{2}-y_{2}+2 \Im\left\langle z_{1}, y_{1}\right\rangle\right|^{2}\right)^{\frac{2 n+2+s p}{4}}},
\end{aligned}
$$

where $\delta_{F}(z, y)=\min _{(x) \in\{z, y\}} d_{F}(x)$, with $d_{F}$ the Carnot-Carathéodory distance from $x$ to $\partial \Omega$. If we choose $F=\partial \Omega$, then we can obtain the inequality with the power $d^{b}$ at the right hand side for the range $b \leq a \frac{p}{q}+\gamma p$.

In general, for a given metric space $X$, our result can be applied for any metric space endowed with the $\alpha$-dimensional Hausdorff measure, as every $\alpha$-Ahlfors-David regular measure is comparable to the $\alpha$-dimensional Hausdorff measure. Also note that our results allow to obtain improved fractional Poincaré-Sobolev inequalities for any ball in a metric measure space satisfying our conditions when the metric of the space is the Carnot-Carathéodory metric, as balls in these spaces are Boman chain domains.

\section{Sufficient conditions for a bounded domain}

In this section we extend Theorem 3.1 in [24] to the more general context of doubling metric measure spaces, improving it by including weights. As an example, we obtain sufficient conditions for a domain in $(X, d, \mu)$ to support the classical improved $(q, p)$-Poincaré inequality. We refer the reader to Remark 1 for the basic definitions concerning chains of balls of a Whitney decomposition of a domain $\Omega$.

First of all, we prove an unweighted fractional $(q, p)$-Poincaré inequality on balls. This lemma was first proved in the Euclidean case in [24, Lemma 2.2].

Lemma 4. Let $B$ be a ball in a metric space $(X, d, \mu)$ with a doubling measure $\mu$. Let $1 \leq q \leq p<\infty$ and let $s, \rho \in(0,1)$. Then,

$$
\begin{aligned}
& \int_{B}\left|u(y)-u_{B}\right|^{q} d \mu(y) \\
& \quad \lesssim \frac{r(B)^{s q}}{\mu(B)^{\frac{q-p}{p}}}\left(\int_{B^{*}} \int_{\left\{z \in B^{*}: d(z, y) \leq \rho r(B)\right\}} \frac{|u(y)-u(z)|^{p} d \mu(z) d \mu(y)}{\mu[B(z, d(z, y))] d(z, y)^{s p}}\right)^{q / p},
\end{aligned}
$$

for any $u \in L_{\mu}^{p}(B)$, where $B^{*}$ is defined as in Remark 1 .

Proof. Let us consider a covering $\mathcal{B}=\left\{B_{i}\right\}_{i \in J}$ of $B$ by $J$ balls of radious $\frac{\rho}{K} r(B)$ for some $K>1$. This can be done in such a way that, when $R$ is the union of two 
balls $B_{i}$ and $B_{j}$ with overlapping dilations (i.e. with $\lambda B_{i} \cap \lambda B_{j} \neq \varnothing$ for some $\lambda>1$ sufficiently small), $R \subset B(y, \rho r(B))$ for every $y \in R$. Also, such an $R$ satisfies $R \subset B^{*}$ and $\mu[B(z, d(z, y))] \lesssim \mu(R)$. Observe that the index set is uniformly finite for every ball $B$, as $X$ is a doubling metric space.

Once we have this construction, observe that, for the union $R$ of two balls in $\mathcal{B}$ with overlapping dilations, we have, by the doubling condition

$$
\begin{aligned}
& \frac{1}{\mu(R)} \int_{R}\left|u(y)-u_{R}\right|^{q} d \mu(y) \\
& \quad \leq\left(\frac{1}{\mu(R)} \int_{R}\left|u(y)-u_{R}\right|^{p} d \mu(y)\right)^{q / p} \\
& \quad \leq\left(\frac{1}{\mu(R)} \int_{R} \frac{1}{\mu(R)} \int_{R}|u(y)-u(z)|^{p} d \mu(z) d \mu(y)\right)^{q / p} \\
& \quad \lesssim\left(\frac{1}{\mu(R)} \int_{R} \int_{R} \frac{|u(y)-u(z)|^{p} \operatorname{diam}(R)^{s p} d \mu(z) d \mu(y)}{\mu[B(z, d(z, y))] d(z, y)^{s p}}\right)^{q / p} \\
& \quad \lesssim \frac{r(B)^{s q}}{\mu(B)^{q / p}}\left(\int_{B^{*}} \int_{B^{*} \cap B(y, \rho r(B))} \frac{|u(y)-u(z)|^{p} d \mu(z) d \mu(y)}{\mu[B(z, d(z, y))] d(z, y)^{s p}}\right)^{q / p}
\end{aligned}
$$

With this in mind, observe that, by Hölder's and Minkowski's,

$$
\begin{aligned}
\frac{1}{\mu(B)} \int_{B}\left|u(y)-u_{B}\right|^{q} d \mu(y) \lesssim & \frac{1}{\mu(B)} \int_{B}\left|u(y)-u_{B_{1}}\right|^{q} d \mu(y) \\
\lesssim & \sum_{j \in J} \frac{1}{\mu\left(B_{j}\right)} \int_{B_{j}}\left|u(y)-u_{B_{j}}\right|^{q} d \mu(y) \\
& +\sum_{j \in J} \frac{1}{\mu\left(B_{j}\right)} \int_{B_{j}}\left|u_{B_{j}}-u_{B_{1}}\right|^{q} d \mu(y) .
\end{aligned}
$$

The first sum is bounded by the quantity above, so it is enough to estimate the second sum. In order to do this, let us fix $B_{j}, j \in J$ and let $\sigma:\{1,2, \ldots, l\} \rightarrow J, l \leq \# J$ an injective map such that $\sigma(1)=1$ and $\sigma(l)=j$, and the subsequent balls $B_{\sigma(i)}$ and $B_{\sigma(i+1)}$ have overlapping dilations. Since $l \leq \# J$, we obtain

$$
\begin{aligned}
\left|u_{B_{j}}-u_{B_{1}}\right|^{q} \leq & \left(\sum_{i=1}^{l-1}\left|u_{B_{\sigma(i+1)}}-u_{B_{\sigma(i)}}\right|\right)^{q} \\
& \lesssim \sum_{i=1}^{l-1}\left|u_{B_{\sigma(i+1)}}-u_{B_{\sigma(i+1)} \cup B_{\sigma(i)}}\right|^{q} \\
& +\sum_{i=1}^{l-1}\left|u_{B_{\sigma(i+1)} \cup B_{\sigma(i)}}-u_{B_{\sigma(i)}}\right|^{q} .
\end{aligned}
$$


The two sums above can be bounded in the same way, so we will just work with the first one. For each term we have

$$
\begin{aligned}
& \left|u_{B_{\sigma(i+1)}}-u_{B_{\sigma(i+1)} \cup B_{\sigma(i)}}\right|^{q} \\
& =\frac{1}{\mu\left(B_{\sigma(i+1)}\right)} \int_{B_{\sigma(i+1)}}\left|u_{B_{\sigma(i+1)}}-u+u-u_{B_{\sigma(i+1)} \cup B_{\sigma(i)}}\right|^{q} d \mu \\
& \quad \lesssim \frac{1}{\mu\left(B_{\sigma(i+1)}\right)} \int_{B_{\sigma(i+1)}}\left|u-u_{B_{\sigma(i+1)}}\right|^{q} d \mu \\
& \quad+\frac{1}{\mu\left(B_{\sigma(i+1)} \cup B_{\sigma(i)}\right)} \int_{B_{\sigma(i+1)} \cup B_{\sigma(i)}} \mid u-u_{\left.B_{\sigma(i+1)} \cup B_{\sigma(i)}\right|^{q}} d \mu,
\end{aligned}
$$

where we have used the conditions on the union of two balls of the covering with nonempty intersection and the doubling condition. In the last two integrals we can apply the first estimate above to obtain the desired result.

With this lemma at hand, we can proceed to the proof of Theorem 4, which gives sufficient conditions on a domain of a doubling metric space to support $\left(w_{\phi}, v_{\Phi, \gamma p}\right)$-improved fractional $(q, p)$-Poincaré inequalities for $q \leq p$ and suitable functions $\phi$ and $\Phi$.

Theorem 4. Let $\Omega$ be a domain in a doubling metric space $(X, d)$ with a Whitney decomposition $W_{M}$ as the one built in Lemma $B$ and with the properties in Remark 1. Let $\phi$ be a positive increasing function satisfying the growth condition $\phi(2 x) \leq C \phi(x)$. Let $1 \leq q \leq p<\infty$, and let $s, \tau \in(0,1)$ and $0 \leq \gamma \leq s$.

(1) If there exists a chain decomposition of $\Omega$ such that

$$
\sum_{E \in W_{M}}\left(\sum_{B \in E\left(W_{M}\right)} r(E)^{(s-\gamma) q} \frac{\phi(r(B))}{\phi(r(E))} \ell\left[\mathcal{C}\left(B^{*}\right)\right]^{q-1} \frac{\mu(B)}{\mu(E)^{q / p}}\right)^{p /(p-q)}<\infty
$$

then $\Omega$ supports the $\left(w_{\phi}, v_{\Phi, \gamma p}\right)$-improved fractional $(q, p)$-Poincaré inequality

$$
\inf _{a \in \mathbb{R}}\|u-a\|_{L^{q}\left(\Omega, w_{\phi} d \mu\right)} \lesssim[u]_{W_{\tau}^{s, p}\left(\Omega, v_{\Phi, \gamma p} d \mu\right)},
$$

where $\Phi(t)=\phi^{\frac{p}{q}}(t)$.

(2) If $q=p$, and if there exists a chain decomposition of $\Omega$ such that

$$
\sup _{E \in W_{M}} \sum_{B \in E\left(W_{M}\right)} r(E)^{(s-\gamma) p} \frac{\phi(r(B))}{\phi(r(E))} \ell\left[\mathcal{C}\left(B^{*}\right)\right]^{p-1} \frac{\mu(B)}{\mu(E)}<\infty,
$$

then $\Omega$ supports the $\left(w_{\phi}, v_{\phi, \gamma p}\right)$-improved fractional $(p, p)$-Poincaré inequality

$$
\inf _{a \in \mathbb{R}}\|u-a\|_{L^{p}\left(\Omega, w_{\phi} d \mu\right)} \lesssim[u]_{W_{\tau}^{s, p}\left(\Omega, v_{\phi, \gamma p} d \mu\right)} .
$$


Proof. We just prove the first statement, as the second one follows in the same way. We can use Hölder's, Minkowski's and the Whitney decomposition of $\Omega$ to obtain

$$
\begin{aligned}
\int_{\Omega}\left|u(x)-u_{B_{0}^{*}}\right| q w_{\phi}(x) d \mu(x) \lesssim & \sum_{B \in W_{M}} \int_{B^{*}}\left|u(x)-u_{B_{0}^{*}}\right|{ }^{q} w_{\phi}(x) d \mu(x) \\
\lesssim & \sum_{B \in W_{M}} \int_{B^{*}}\left|u(x)-u_{B^{*}}\right|{ }^{q} w_{\phi}(x) d \mu(x) \\
& +\sum_{B \in W_{M}} \int_{B^{*}}\left|u_{B^{*}}-u_{B_{0}^{*}}\right|{ }^{q} w_{\phi}(x) d \mu(x) .
\end{aligned}
$$

We begin by estimating the first sum. Using Lemma 4 with $\rho=C_{M} \tau$ (where $C_{M}<1$ is such that $\rho r\left(B^{*}\right) \leq \tau d(x)$ for any $\left.x \in B^{* *}\right)$ and the fact that $d(x) \asymp r\left(B^{*}\right)$ for any $x \in B^{*}$ (and the corresponding fact for $B^{* *}$ ), then, taking into account the choice of $M$ and the growth condition on $\phi$, we obtain

$$
\int_{B^{*}}\left|u(x)-u_{B^{*}}\right|{ }^{q} w_{\phi}(x) d \mu(x) \lesssim \frac{r(B)^{(s-\gamma) q}}{\mu\left(B^{*}\right)^{q / p-1}}[u]_{W_{\tau}^{s, p}\left(B^{* *}, w_{\Phi, \gamma p}\right)}^{q} .
$$

Thus,

$$
\begin{aligned}
& \sum_{B \in W_{M}} \int_{B^{*}}\left|u(x)-u_{B^{*}}\right|^{q} w_{\phi}(x) d \mu(x) \\
& \lesssim \sum_{B \in W_{M}} \frac{r(B)^{(s-\gamma) q}}{\mu\left(B^{*}\right)^{q / p-1}}[u]_{W_{\tau}^{s, p}\left(B^{* *}, w_{\Phi, \gamma p}\right)}^{q} \\
& \leq\left(\sum_{B \in W_{M}} \mu\left(B^{*}\right)\right)^{\frac{p-q}{p}}\left(\sum_{B \in W_{M}} r(B)^{(s-\gamma) p}[u]_{W_{\tau}^{s, p}\left(B^{* *}, w_{\Phi, \gamma p}\right)}^{p}\right)^{q / p} \\
& \lesssim \mu(\Omega)^{\frac{p-q}{p}}\left(\sum_{B \in W_{M}} r(B)^{(s-\gamma) p}[u]_{W_{\tau}^{s, p}\left(B^{* *}, w_{\Phi, \gamma p}\right)}^{p / p}\right)^{q / p} \\
& \lesssim \mu(\Omega)^{\frac{p-q}{p}} \operatorname{diam}(\Omega)^{(s-\gamma) q}[u]_{W_{\tau}^{s, p}\left(\Omega, v_{\Phi, \gamma p}\right)}^{q}
\end{aligned}
$$

where we have used that $\left\{B^{*}\right\}_{B \in W_{M}}$ and $\left\{B^{* *}\right\}_{B \in W_{M}}$ are families with uniformly bounded overlapping contained in $\Omega$ and also that, in the domain of integration, the distance from each variable to the boundary of $\Omega$ is comparable to the other. 
Next, we estimate the second sum in (5.4). By using chains of the decomposition and again that $d(x) \asymp r\left(B^{*}\right), x \in B^{*}$,

$$
\begin{aligned}
& \sum_{B \in W_{M}} \int_{B^{*}}\left|u_{B^{*}}-u_{B_{0}^{*}}\right|^{q} w_{\phi}(x) d \mu(x) \\
& \lesssim \sum_{B \in W_{M}} \mu(B) \phi(r(B))\left(\sum_{j=1}^{k}\left|u_{B_{j}^{*}}-u_{B_{j-1}^{*}}\right|\right)^{q} \\
& \leq \sum_{B \in W_{M}} \ell\left[\mathcal{C}\left(B^{*}\right)\right]^{q-1} \mu(B) \phi(r(B))\left(\sum_{j=1}^{k}\left|u_{B_{j}^{*}}-u_{B_{j-1}^{*}}\right|^{q}\right) .
\end{aligned}
$$

Since $\max \left\{\mu\left(B_{j}^{*}\right), \mu\left(B_{j-1}^{*}\right)\right\} \lesssim \mu\left(B_{j}^{*} \cap B_{j-1}^{*}\right)$ (see (2.10)), we can write, by using Hölder's inequality,

$$
\begin{aligned}
\left|u_{B_{j}^{*}}-u_{B_{j-1}^{*}}\right|^{q} & \lesssim \sum_{i=j-1}^{j}\left(\mu\left(B_{i}^{*}\right)^{-1} \int_{B_{i}^{*}}\left|u(x)-u_{B_{i}^{*}}\right| d \mu(x)\right)^{q} \\
& \leq \sum_{i=j-1}^{j} \mu\left(B_{i}^{*}\right)^{-1} \int_{B_{i}^{*}}\left|u(x)-u_{B_{i}^{*}}\right|^{q} d \mu(x),
\end{aligned}
$$

where, for the first inequality, we have used that

$$
\begin{aligned}
\left|u_{B_{j}^{*}}-u_{B_{j-1}^{*}}\right|^{q} & =\left|\frac{1}{\mu\left(B_{j}^{*} \cap B_{j-1}^{*}\right)} \int_{B_{j}^{*} \cap B_{j-1}^{*}}\left(u_{B_{j}^{*}}-u(x)+u(x)-u_{B_{j-1}^{*}}\right) d \mu(x)\right|^{q} \\
& \lesssim \sum_{i=j-1}^{j} \frac{1}{\mu\left(B_{j}^{*} \cap B_{j-1}^{*}\right)} \int_{B_{j}^{*} \cap B_{j-1}^{*}}\left|u_{B_{i}^{*}}-u(x)\right|^{q} .
\end{aligned}
$$

A new application of Lemma 4 gives

$$
\left|u_{B_{j}^{*}}-u_{B_{j-1}^{*}}\right|^{q} \lesssim \sum_{i=j-1}^{j} \frac{r\left(B_{i}\right)^{(s-\gamma) q}}{\phi\left(r\left(B_{i}\right)\right)} \mu\left(B_{i}^{*}\right)^{-q / p}[u]_{W_{\tau}^{s, p}\left(B_{i}^{* *}, w_{\Phi, \gamma p}\right)}^{q},
$$

so the second sum in (5.4) is bounded by the sum

$$
\sum_{B \in W_{M}} \phi(r(B)) \ell\left[\mathcal{C}\left(B^{*}\right)\right]^{q-1} \mu(B)\left[\sum_{j=0}^{k} \frac{r\left(B_{j}\right)^{(s-\gamma) q}}{\phi\left(r\left(B_{j}\right)\right)} \mu\left(B_{j}^{*}\right)^{-q / p}[u]_{W_{\tau}^{s, p}\left(B_{j}^{* *}, w_{\Phi, \gamma p}\right)}^{q}\right] .
$$


Rearranging the sum as in [24], we get

$$
\begin{aligned}
& \sum_{B \in W_{M}} \int_{B^{*}}\left|u_{B^{*}}-u_{B_{0}^{*}}\right|^{q} d \mu(x) \\
& \lesssim \sum_{E \in W_{M}} \sum_{B \in E\left(W_{M}\right)} r(E)^{(s-\gamma) q} \frac{\phi(r(B))}{\phi(r(E))} \ell\left[\mathcal{C}\left(B^{*}\right)\right]^{q-1} \frac{\mu(B)}{\mu(E)^{q / p}}[u]_{W_{\tau}^{s, p}\left(E^{* *}, w_{\Phi, \gamma p}\right)}^{q}
\end{aligned}
$$

Now, Hölder's inequality together with the hypothesis allow us to bound the sum above by $[u]_{W_{\tau}^{s, p}\left(\Omega, v_{\Phi, \gamma p}\right)}^{q}$ times the following expresion

$$
\left[\sum_{E \in W_{M}}\left(\sum_{B \in E\left(W_{M}\right)} r(E)^{(s-\gamma) q} \frac{\phi(r(B))}{\phi(r(E))} \ell\left[\mathcal{C}\left(B^{*}\right)\right]^{q-1} \frac{\mu(B)}{\mu(E)^{q / p}}\right)^{\frac{p}{p-q}}\right]^{\frac{p-q}{p}},
$$

and the result follows.

Remark 7. Observe that, in the case $0 \leq \gamma<s$, the constant in the obtained Poincaré inequality depends on the size of the domain $\Omega$. This also happens if one thinks of a nonimproved version of the result, as one can check in the proof of Theorem 3.1 in [24].

\section{An example of application of Theorem 4: the case of John domains in a complete metric space}

In this section we will prove a positive result for John domains in complete doubling metric spaces. We begin with a generalization of [24, Lemma 2.7]. To this end, we will use the dyadic structure given by Hytönen and Kairema and introduced in Section 2. We will also use the concept of porous sets in a metric space.

Definition 2. A set $S$ in a metric space $(X, d)$ is porous in $X$ if for some $\varkappa \in(0,1]$ the following statement is true: for every $x \in X$ and $0<r \leq 1$ there is $y \in B(x, r)$ such that $B(y, \varkappa r) \cap S=\varnothing$.

Examples of porous sets are the boundaries of bounded John domains in a complete metric space (see [38] for the result in the Euclidean case and observe that Ascoli-Arzela's theorem allows us to prove the result in a complete metric space).

Lemma 5. Let $(X, d, \mu)$ be a metric space endowed with a doubling measure, and let $S \subset X$ be a porous set. Let $1 \leq p<\infty$. If $x \in S$ and $0<r \leq 1$, then

$$
\int_{B(x, r)} \log ^{p} \frac{1}{d(y, S)} d \mu(y) \leq c \mu(B(x, r))\left(1+\log ^{p} \frac{1}{r}\right),
$$

where the constant $c$ is independent of $x$ and $r$. 
Proof. We follow here the notations of Theorem A. Let us define

$$
\mathcal{C}_{S, \gamma}:=\left\{Q \in \mathcal{D}: \gamma^{-1} d\left(z_{Q}, S\right) \leq r(b(Q)) \leq r(B(Q)) \leq 1\right\},
$$

where $z_{Q}$ is the center of the two balls $b(Q)$ and $B(Q)$ associated to the dyadic cube $Q$.

Suppose $R \in \mathcal{D}$ is a dyadic cube such that $r(B(R)) \leq 1$ and such that $d(y, S) \leq$ $2\left(\frac{C_{1}}{c_{1} s}\right)^{2} r(b(R))$ for some $y \in R$. Then,

$$
\begin{aligned}
d\left(z_{R}, S\right) & \leq d\left(z_{R}, y\right)+d(y, S) \leq r(B(R))+2\left(\frac{C_{1}}{c_{1} s}\right)^{2} r(b(R)) \\
& \leq \frac{C_{1}}{c_{1}} r(b(R))+2\left(\frac{C_{1}}{c_{1} s}\right)^{2} r(b(R))=\left(\frac{C_{1}}{c_{1}}+2\left(\frac{C_{1}}{c_{1} s}\right)^{2}\right) r(b(R)),
\end{aligned}
$$

so we have $R \in \mathcal{C}_{S, \gamma}$ for $\gamma=\frac{C_{1}}{c_{1}}+2\left(\frac{C_{1}}{c_{1} s}\right)^{2}$.

Fix $j$ a nonnegative integer such that $C_{1} s^{j+1} \leq r<C_{1} s^{j}$, and consider a dyadic cube $Q \in \mathcal{D}_{j+1}$ for which $Q \cap B(x, r) \neq \varnothing$. We can cover $B(x, r)$ with cubes like this, as $\mathcal{D}_{j+1}$ partitions $X$, so it will be enough to prove that, for any of these cubes $Q$, we can get

$$
\left\|\log \frac{1}{d(\cdot, S)}\right\|_{L_{\mu}^{p}(Q \cap B(x, r))}^{p} \lesssim \mu(Q \cap B(x, r))\left(1+\log ^{p} \frac{1}{r}\right),
$$

as we are working in a doubling metric space, which implies that the size of any covering of $B$ by cubes of the size stated above, is uniformly bounded.

By the porosity of $S$ we know that, since $\mu$ is doubling, $S$ has zero $\mu$-measure [30, Proposition 3.4], so it is enough to consider points $y \in Q \cap B(x, r) \backslash S$. Since $x \in S$, we have that

$$
d(y, S) \leq d(y, x) \leq r \leq C_{1} s^{j} \leq C_{1}\left(c_{1} s\right)^{-1} \operatorname{diam} Q
$$

i.e.,

$$
1 \leq C_{1}\left(c_{1} s\right)^{-1} \frac{\operatorname{diam} Q}{d(y, S)}
$$

Consider now a sequence of dyadic cubes $Q=Q_{0}(y) \supset Q_{1}(y) \supset \ldots \supset Q_{m}(y)$, each of them containing $y$ and $Q_{i}(y)$ and $Q_{i+1}(y)$ being immediate ancestor and son, respectively. This, in particular, means that

$$
\frac{2 C_{1}}{c_{1}} s^{-1} \geq \frac{\operatorname{diam} B\left(Q_{i}(y)\right)}{\operatorname{diam} b\left(Q_{i+1}(y)\right)} \geq \frac{\operatorname{diam} Q_{i}(y)}{\operatorname{diam} Q_{i+1}(y)} \geq \frac{\operatorname{diam} b\left(Q_{i}(y)\right)}{\operatorname{diam} B\left(Q_{i+1}(y)\right)} \geq \frac{c_{1}}{2 C_{1}} s^{-1}
$$


We choose $m$ such that the last cube in the sequence satisfies

$$
\frac{c_{1} s^{2}}{C_{1}} d(y, S) \leq \operatorname{diam} Q_{m}(y)<\frac{c_{1} s}{C_{1}} d(y, S) .
$$

From (6.2) it follows that $m \geq 1$, and by (6.3) and (6.4),

$$
\left(\frac{2 C_{1}}{c_{1}} s^{-1}\right)^{m} \geq \prod_{i=0}^{m-1} \frac{\operatorname{diam} Q_{i}(y)}{\operatorname{diam} Q_{i+1}(y)}=\frac{\operatorname{diam} Q_{0}(y)}{\operatorname{diam} Q_{m}(y)}>C_{1}\left(c_{1} s\right)^{-1} \frac{\operatorname{diam} Q}{d(y, S)} \geq 1 .
$$

Thus,

$$
\begin{aligned}
m & \geq \log _{\frac{2 C_{1}}{c_{1} s}}\left(\frac{2 C_{1}}{c_{1} s}\right)^{m}=\frac{1}{\log \left(\frac{2 C_{1}}{c_{1} s}\right)} \log \left(\frac{2 C_{1}}{c_{1} s}\right)^{m} \\
& \geq \frac{1}{\log \left(\frac{2 C_{1}}{c_{1} s}\right)}\left[\log \frac{C_{1} \operatorname{diam} Q}{c_{1} s}-\log d(y, S)\right] \geq 0 .
\end{aligned}
$$

Furthermore, (6.4) and (6.1) yield $Q_{i}(y) \in \mathcal{C}_{S, \gamma}$ for $i=0,1, \ldots, m$ when $\gamma$ is as in (6.1). Thus, we obtain

$$
\begin{aligned}
\sum_{\substack{R \in \mathcal{C}_{S, \gamma} \\
R \subset Q}} \chi_{R}(y) & \geq 1+m \geq 1+\frac{1}{\log \left(\frac{2 C_{1}}{c_{1} s}\right)}\left[\log \frac{C_{1} \operatorname{diam} Q}{c_{1} s}-\log d(y, S)\right] \\
& \geq \frac{1}{\log \left(\frac{2 C_{1}}{c_{1} s}\right)}\left[\log \left(\frac{2 C_{1}}{c_{1} s}\right)+\log \frac{C_{1} \operatorname{diam} Q}{c_{1} s}-\log d(y, S)\right] \geq 0,
\end{aligned}
$$

as $2 C_{1}>s c_{1}$.

If we now integrate and apply triangle inequality, we get

$$
\begin{aligned}
& \left.\left\|\log \frac{1}{d(\cdot, S)}\right\|\right|_{L_{\mu}^{p}(Q \cap B(x, r))} \\
& \leq\left|\log \left(\frac{2 C_{1}^{2} \operatorname{diam} Q}{\left(c_{1} s\right)^{2}}\right)\right| \mu(Q \cap B(x, r))^{\frac{1}{p}}+\log \left(\frac{2 C_{1}}{c_{1} s}\right)\left\|\sum_{R \in \mathcal{C}_{S, \gamma}} \chi_{R}\right\| \|_{L_{\mu}^{p}(Q \cap B(x, r))} \\
& \lesssim\left(1+\log \frac{C_{1}}{c_{1} s \operatorname{diam} Q}\right) \mu(Q \cap B(x, r))^{\frac{1}{p}}+\left\|\sum_{R \in \mathcal{C}_{S, \gamma}} \chi_{R}\right\|_{R \subset Q}\|\|_{L_{\mu}^{p}(Q \cap B(x, r))} \chi_{\substack{R \in \mathcal{C}_{S, \gamma} \\
R \subset Q}} \chi_{C_{R}} \|_{L_{\mu}^{p}(Q \cap B(x, r))},
\end{aligned}
$$


where we have used that $2 C_{1}>e c_{1} s$ (this is immediate by taking into account the definitions of $C_{1}, c_{1}$ and $s$ in Theorem A), and the fact that, in particular, $\operatorname{diam} Q \leq$ $C_{1} s^{j+1} \leq r<1$. Now observe that

$$
\operatorname{diam} Q \geq c_{1} s^{j+1}=\frac{c_{1} s}{C_{1}} C_{1} s^{j}>\frac{c_{1} s}{C_{1}} r
$$

So

$$
\begin{aligned}
& \left\|\log \frac{1}{d(\cdot, S)}\right\|_{L_{\mu}^{p}(Q \cap B(x, r))} \\
& \quad \lesssim\left(1+\log \frac{1}{r}\right) \mu(Q \cap B(x, r))^{1 / p}+\left\|\sum_{\substack{R \in \mathcal{C}_{S, \gamma} \\
R \subset Q}} \chi_{R}\right\|_{L_{\mu}^{p}(Q \cap B(x, r))} .
\end{aligned}
$$

Since $S$ is porous in $X$, we can follow the proof of [27, Theorem 2.10] in our context. We obtain a finite positive constant $K_{\varkappa}$, depending on the porosity constant $\varkappa$, and families

$$
\{\widehat{R}\}_{R \in \mathcal{C}_{S, \gamma}^{k}}, \quad \mathcal{C}_{S, \gamma}^{k} \subset \mathcal{C}_{S, \gamma}, \quad k=0,1, \ldots, K_{\varkappa}-1
$$

where each $\{\widehat{R}\}_{R \in \mathcal{C}_{S, \gamma}^{k}}$ is a disjoint family of cubes $\widehat{R} \subset R$, such that

$$
\begin{aligned}
\left\|\sum_{\substack{R \in \mathcal{C}_{S, \gamma} \\
R \subset Q}} \chi_{R}\right\|_{L_{\mu}^{p}(Q \cap B(x, r))} & \lesssim \sum_{k=0}^{K_{\varkappa}-1}\left\|\sum_{\substack{R \in \mathcal{C}_{S, \gamma}^{k} \\
R \subset Q}} \chi_{\widehat{R}}\right\|_{L_{\mu}^{p}(Q \cap B(x, r))} \\
& \leq \sum_{k=0}^{K_{\varkappa}-1}\left\|\chi_{Q}\right\|_{L_{\mu}^{p}(Q \cap B(x, r))} \lesssim \mu(Q \cap B(x, r))^{1 / p} .
\end{aligned}
$$

Hence, we get

$$
\left\|\log \frac{1}{d(\cdot, S)}\right\|_{L_{\mu}^{p}(Q \cap B(x, r))} \lesssim\left(1+\log \frac{1}{r}\right) \mu(Q \cap B(x, r))^{1 / p},
$$

which finishes the proof.

Following [24] it is possible to construct a chain decomposition of a given John domain $\Omega$ on a metric space in such a way that, for a given ball $B \in W_{M}$ in a Whitney covering of the domain, we have that the chain associated to $B$ satisfies

$$
\ell\left[\mathcal{C}\left(B^{*}\right)\right] \lesssim\left(1+\log \frac{1}{\operatorname{diam} B}\right)
$$


Using this result and the fact that a John domain in a complete metric doubling space has porous boundary, we can prove an $\left(w_{\phi}, v_{\phi, \gamma p}\right)$-improved fractional $(p, p)$-Poincaré inequality on John domains.

Theorem 5. Let $1 \leq p<\infty, \tau, s \in(0,1)$ and $0 \leq \gamma<s$. Let $\phi$ be a positive increasing function and define $w_{\phi}$ and $v_{\phi, \gamma p}$ as in Theorem 4 . A John domain $\Omega$ in a complete doubling metric space $(X, d, \mu)$ supports the $\left(w_{\phi}, v_{\phi, \gamma p}\right)$-improved fractional $(p, p)$-Poincaré inequality.

Proof. We may assume $\operatorname{diam} \Omega \leq 1$. We will check condition (5.3) of Theorem 4. If $E$ is a ball in $W_{M}$, then

$$
\bigcup_{B \in E\left(W_{M}\right)} B \subset B\left(\omega_{E}, \min \{1, c \operatorname{diam}(E)\}\right),
$$

where $\omega_{E}$ is the closest point in $\partial \Omega$ to $x_{E}$ and $c$ is a positive constant independent of $E$. This follows from the fact that, if $B \in E\left(W_{M}\right)$, then $E$ is closer to $x_{0}$ than $B$, so $B$ is closer to $\partial \Omega$ than $E$ (recall that diam $E \asymp d\left(x_{E}, \partial \Omega\right)$ ).

Using this and (6.6), we obtain

$$
\begin{aligned}
& \sum_{B \in E\left(W_{M}\right)} \phi(r(B)) \ell\left[\mathcal{C}\left(B^{*}\right)\right]^{p-1} \mu(B) \\
& \lesssim \sum_{B \in E\left(W_{M}\right)} \phi(r(B)) \mu(B)\left(1+\log \frac{1}{\operatorname{diam} B}\right)^{p-1} \\
& \lesssim \sum_{B \in E\left(W_{M}\right)} \phi(r(B)) \mu(B)\left(1+\log ^{p} \frac{1}{\operatorname{diam} B}\right) \\
& \lesssim \sum_{B \in E\left(W_{M}\right)} \int_{B} \phi(r(B))\left(1+\log ^{p} \frac{1}{d(y, \partial \Omega)}\right) d \mu(y) \\
& \lesssim \int_{B\left(\omega_{E}, \min \{1, c \operatorname{diam} E\}\right)} \phi(r(E))\left(1+\log ^{p} \frac{1}{d(y, \partial \Omega)}\right) d \mu(y)
\end{aligned}
$$

Since $X$ is a complete space, the boundary of the John domain $\Omega$ is porous in $S$, so we can apply Lemma 5 to $\omega_{E}$ with $r=\min \{1, c \operatorname{diam} E\}$ in order to obtain

$$
\begin{aligned}
& \sum_{B \in E\left(W_{M}\right)} \phi(r(B)) \ell\left[\mathcal{C}\left(B^{*}\right)\right]^{p-1} \mu(B) \\
& \lesssim \phi(r(E)) \mu\left[B\left(\omega_{E}, \min \{1, c \operatorname{diam} E\}\right)\right]\left(1+\log ^{p} \frac{1}{\min \{1, c \operatorname{diam} E\}}\right)
\end{aligned}
$$


Thus, we can check (5.3) for $\Omega$, obtaining

$$
\begin{aligned}
& \sup _{E \in W_{M}} \sum_{B \in E\left(W_{M}\right)} \frac{\phi(r(B))}{\phi(r(E))} \ell\left[\mathcal{C}\left(B^{*}\right)\right]^{p-1} r(E)^{(s-\gamma) p} \frac{\mu(B)}{\mu(E)} \\
& \lesssim \sup _{E \in W_{M}} \frac{\phi(r(E))}{\phi(r(E))} r(E)^{(s-\gamma) p}\left(1+\log ^{p} \frac{1}{\operatorname{diam} E}\right) \frac{\mu\left[B\left(\omega_{E}, \min \{1, c \operatorname{diam} E\}\right)\right]}{\mu(E)} \\
& \lesssim \sup _{E \in W_{M}} r(E)^{(s-\gamma) p}\left(1+\log ^{p} \frac{1}{\operatorname{diam} E}\right)\left(\frac{\min \{1,2 c r(E)\}}{r(E)}\right)^{n_{\mu}} \\
& <\infty,
\end{aligned}
$$

where we have used the doubling condition on $\mu$ and the fact that the ball $E$ is in the ball $B\left(\omega_{E}, \min \{1, c \operatorname{diam} E\}\right)$. This last sum is finite as $1+\log ^{p} \frac{1}{t} \lesssim \frac{1}{t^{\eta p}}$, for $0<\eta<s-\gamma$ if $t<1$.

Remark 8. The growth condition on $\phi$ is not necessary. Moreover, the result also holds for a function $\phi$ satisfying $\phi(2 t) \leq t^{\delta} \phi(t)$, where $\delta>\gamma-s$ (the case $\gamma=s$ is allowed here).

Also, it is interesting to compare this result with Theorem 2 in the sense that here we just need $\mu$ to be a doubling measure in order to get the inequality, whereas in Theorem 2 one asks $\mu$ to be a more regular measure.

Acknowledgements. The first and second authors are supported by ANPCyT under grant PICT 2014-1771. The first author is also supported by Universidad Nacional de La Plata under grant 11/X805, and the second one by Universidad de Buenos Aires under grant 20020120100050BA.

The third author is supported by the Basque Government through the BERC 2018-2021 program and by Spanish Ministry of Science, Innovation and Universities through BCAM Severo Ochoa accreditation SEV-2017-0718. He is also supported by MINECO through the MTM2017-82160-C2-1-P project funded by (AEI/FEDER, UE), acronym "HAQMEC" and through "la Caixa" grant. This work was initiated during the visit of the third author to M. E. Cejas and I. Drelichman at Universidad Nacional de La Plata (UNLP) and Universidad de Buenos Aires (UBA) in December 2017. The third author is very grateful for the kind hospitality from people from UNLP and UBA and he also wants to thank IMAS (CONICET \& UBA) for its support for the author's stay at Buenos Aires. This work was finished during a stay of the first author at the BCAM-Basque Center for Applied Mathematics in 2018 supported both by CONICET and BCAM-Basque Center for Applied Mathematics.

Recently, after sending the paper for publication and while working in some extensions of the results here through a different approach, the third author knew through personal communications about the existence of the work [6] by Seng Kee 
Chua. In this paper, the author provides (among other things) a quite general method for passing from a local inequality to a global one. This new method allows to consider more general measures than the doubling ones, once we know a starting point on balls.

Also, we want to thank the referee for important comments about the introduction and for pointing us some misprints and missing references.

\section{References}

1. Acosta, G., Cejas, E. and Duran, R., Improved Poincaré inequalities and solutions of the divergence in weighted norms, Ann. Acad. Sci. Fenn. Math. 42 (2017), 211-226. MR3558524

2. Boas, H. P. and Straube, E. J., Integral Inequalities of Hardy and Poincaré Type, Proc. Amer. Math. Soc. 103 (1988), 172-176. MR0938664

3. Bogdan, B., Remarks on Sobolev imbedding inequalities, in Lecture Notes in Mathematics 1351, pp. 52-68, Springer, Berlin, Heidelberg, 1988. MR0982072

4. Boman, J., L ${ }^{p}$-estimates for Very Strongly Elliptic Systems. Reports: Matematiska Institutionen, University of Stockholm, Sweden, 1982.

5. Buckley, S. and Koskela, P., Sobolev-Poincaré implies John, Math. Res. Lett. 2 (1995), 577-593. MR1359964

6. Chus, S.-K., Embedding and compact embedding for weighted and abstract Sobolev spaces. To appear in Pacific J. Math. (2018).

7. Chua, S.-K., Weighted Sobolev Inequalities on Domains Satisfying the Chain Condition, Proceedings of the American Mathematical Society 117 (1993), 449457. MR1140667

8. Coifman, R. R. and Weiss, G., Analyse Harmonique Non-Commutative sur Certains Espaces Homogenes, Lecture Notes in Mathematics 242, Springer, Berlin, 1971. MR0499948

9. Drelichman, I. and Durán, R. G., On the interpolation space $\left(L^{P}(Q), W^{1, P}(Q)\right)_{s, p}$ in non-smooth domains, J. Math. Anal. Appl. 470 (2019), 91-101. MR3865123

10. Drelichman, I. and Durán, R., Improved Poincaré inequalities in fractional Sobolev spaces, Ann. Acad. Sci. Fenn. Math. 43 (2017), 885-903. MR3839841

11. Drelichman, I. and Durán, R., Improved Poincaré inequality with weights, J. Math. Anal. Appl. 347 (2008), 286-293. MR2433844

12. Dyda, B., On comparability of integral forms, J. Math. Anal. Appl. 318 (2006), 564577. MR2215170

13. Dyda, B., Ihnatsyeva, L. and VÄhäkangas, A., On improved fractional SobolevPoincaré inequalities, Ark. Mat. 54 (2016), 437-454. MR3546360

14. Dyda, B. and VÄHÄKangas, A. V., Characterizations for fractional Hardy inequality, Adv. Cal. Var. 8 (2015), 173-182. MR3331699

15. Franchi, B., Pérez, C. and Wheeden, R. L., Self-improving properties of JohnNirenberg and Poincaré inequalities on spaces of homogeneous type, J. Funct. Anal. 153 (1998), 108-146. MR1609261

16. Garcia-Cuerva, J. and Gatto, A. E., Boundedness properties of fractional integral operators associated to non-doubling measures, Stud. Math. 162 
(2003). MR2047654

17. Gogatishvili, A., Koskela, P. and Shanmugalingam, N., Interpolation properties of Besov spaces defined on metric spaces, Math. Nachr. 283 (2010), 215231. MR2604119

18. HajŁasz, P., Sobolev inequalities, truncation method, and John domains, Pap. Anal. Rep. Univ. Jyv. Dept. Math. Statist. 83 (2001), 109-126. MR1886617

19. HajŁasz, P., Sobolev spaces on an arbitrary metric space, Potential Anal. 5 (1996), 403-415. MR1401074

20. HajŁasz, P. and Koskela, P., Sobolev Met Poincaré, in Memoirs AMS 688, Amer. Mathematical Society, Providence, 2000. MR1683160

21. Heinonen, J., Lectures on Analysis on Metric Spaces, Universitext, Springer, New York, 2001. MR1800917

22. Heinonen, J., Koskela, P., Shanmugalingam, N. and Tyson, J. T., Sobolev Spaces on Metric Measure Spaces: An Approach Based on Upper Gradients. New Mathematical Monographs, Cambridge University Press, Cambridge, 2015. MR3363168

23. Hurri-Syrjänen, R., An Improved Poincaré Inequality, Proc. Amer. Math. Soc. 120 (1994), 213-222. MR1169032

24. Hurri-Syrjänen, R. and VÄhäkAngas, A., On fractional Poincaré inequalities, $J$. Anal. Math. 120 (2013), 85-104. MR3095149

25. Hurri, R., The weighted Poincaré inequalities, Math. Scand. 67 (1990), 145160. MR1081294

26. Hytönen, T. and Kairema, A., Systems of dyadic cubes in a doubling metric space, Colloq. Math. 126 (2012), 1-33. MR2901199

27. Ihnatsyeva, L. and VäHÄKangas, A. V., Characterization of traces of smooth functions on Ahlfors regular sets, J. Funct. Anal. 265 (2013), 18701915. MR3084491

28. Hedberg, L. I., On Certain Convolution Inequalities, Proc. Amer. Math. Soc. 36 (1972), 505. MR0312232

29. IwANiEc, T. and Nolder, C. A., Hardy-Littlewood inequality for quasiregular mappings in certain domains in $\mathbb{R}^{n}$, Ann. Acad. Sci. Fenn. Ser. A. I. Math. 10 (1985), 267-282. MR0802488

30. Järvenpä̈̈, E., JÄrvenpä̈̈, M., KÄenmäki, A., Rajala, T., Rogovin, S. and Suomala, V., Packing dimension and Ahlfors regularity of porous sets in metric spaces, Math. Z. 266 (2010), 83-105. MR2670673

31. Jerison, D., The Poincaré inequality for vector fields satisfying Hormander's condition, Duke Math. J. 53 (1986), 503-523. MR0850547

32. Fritz, J., Rotation and strain, Comm. Pure Appl. Math. 14 (1961), 391413. MR0138225

33. Jost, J., Partial Differential Equations, Graduate Texts in Mathematics, Springer, New York, 2007. MR2302683

34. López García, F. and Hurri-Syrjanen, R., On the weighted fractional Poincarétype inequalities Colloq. Math. 157 (2019), 213-230. MR3984270

35. Macmanus, P. and Perez, C., Generalized Poincaré inequalities: sharp self-improving properties, Internat. Math. Res. Notices 2 (1998), 101116. MR1604816 
36. Macías, R. A. and Segovia, C., A Decomposition into Atoms of Distributions on Spaces of Homogeneous Type, Adv. Math. 33, 271309 (1979). MR0546296

37. Olli, M. and Sarvas, J., Injectivity theorems in plane and space, Ann. Acad. Sci. Fenn. Math Ser. A. I. Math. 4 (1978/1979), 383-401. MR0565886

38. Olli, M. and Vuorinen, M., Whitney cubes, p-capacity, and Minkowski content. University of Jyvaskyla, Department of Mathematics, 1985. MR0880256

39. MaZ'YA, V., Sobolev Spaces: with Applications to Elliptic Partial Differential Equations, 2nd ed., Grundlehren der mathematischen Wissenschaften 342, Springer, Berlin, Heidelberg, 2011. MR2777530

40. Ojala, T., Rajala, T. and Suomala, V., Thin and fat sets for doubling measures in metric spaces, Stud. Math. 208 (2012), 195-211. MR2911493

41. PÉrez, C. and Rela, E., Degenerate Poincaré-Sobolev inequalities. To appear in Trans. Amer. Math. Soc. (2018). DOI: https://doi.org/10.1090/tran/7775.

42. Pietruska-Paluba, K., Heat kernels on metric spaces and a characterization of constant functions, Manuscripta Math. 115 (2004), 389-399. MR2102059

43. Reshetnyak, Y. G., Integral representations of differentiable functions in domains with nonsmooth boundary, Sib. Math. J. 21 (1980), 833-839. MR0601195

44. Sawyer, E. and Wheeden, R. L., Weighted Inequalities for Fractional Integrals on Euclidean and Homogeneous Spaces, Amer. J. Math. 114 (1992), 813874. MR1175693

45. Stein, E. M. (ed.), Beijing Lectures in Harmonic Analysis, Annals of Mathematics Studies 112, Princeton University Press, Princeton, 1986. MR1232192

46. WheEden, R. L., A characterization of some weighted norm inequalities for the fractional maximal function, Studia Math 107 (1993), 257-272. MR1247202

47. YAng, D., Real interpolations for Besov and Triebel-Lizorkin spaces on spaces of homogeneous type, Math. Nachr. 273 (2004), 96-113. MR2084959

María Eugenia Cejas

Departmento de Matemática

Universidad Nacional de La Plata, 1900,

CONICET

La Plata

Argentina

ecejas@mate.unlp.edu.ar
Irene Drelichman

IMAS (UBA-CONICET), Facultad de Ciencias Exactas y Naturales

Universidad de Buenos Aires

Ciudad Universitaria

1428 Buenos Aires

Argentina

irene@drelichman.com

Javier C. Martínez-Perales

BCAM-Basque Center for Applied Math-

ematics

48009 Bilbao

Spain

jmartinez@bcamath.org

Received February 20, 2019

in revised form July 4, 2019 